\title{
Accuracy of analytical models of the large-scale matter distribution
}

\author{
Patrick Valageas \\ Institut de Physique Théorique, \\ CEA, IPhT, F-91191 Gif-sur-Yvette, Cédex, France \\ CNRS, URA 2306, F-91191 Gif-sur-Yvette, Cédex, France
}

(Dated: January 28, 2021)

\begin{abstract}
We investigate the possible accuracy that can be reached by analytical models for the matter density power spectrum and correlation function. Using a realistic description of the power spectrum that combines perturbation theory with a halo model, we study the convergence rate of several perturbative expansion schemes and the impact of nonperturbative effects, as well as the sensitivity to phenomenological halo parameters. We check that the simple reorganization of the standard perturbative expansion, with a Gaussian damping prefactor, provides a well-ordered convergence and a finite correlation function that yields a percent accuracy at the baryon acoustic oscillation peak (as soon as one goes to second order). Lagrangian-space expansions are somewhat more efficient, when truncated at low orders, but may diverge at high orders. We find that whereas the uncertainty on the halo-profile mass-concentration relation is not a strong limitation, the uncertainty on the halo mass function can severely limit the accuracy of theoretical predictions for $P(k)$ (this also applies to the power spectra measured in numerical simulations). The real-space correlation function provides a better separation between perturbative and nonperturbative effects, which are restricted to $x \lesssim 10 h^{-1} \mathrm{Mpc}$ at all redshifts.

PACS numbers: 98.80.-k, 98.65.Dx
\end{abstract}

\section{INTRODUCTION}

The growth of large-scale structures in the Universe through gravitational instability is a key ingredient of modern cosmology [1] and an important probe of cosmological parameters. In particular, future galaxy surveys aim at a percent precision on a broad range of scales to constrain the dark energy component [2]. On large scales or at high redshifts, where the amplitude of the density fluctuations is small, it is sufficient to use linear theory, whereas on small scales, in the highly nonlinear regime, one must use numerical simulations or phenomenological models, such as the halo model [3], which are also calibrated on simulations. On intermediate scales, which are the focus of several observational probes, such as measures of baryon acoustic oscillations (BAOs) [4, 5], perturbative approaches provide systematic methods to go beyond linear theory and increase the range of accurate theoretical predictions.

This has led to a renewed interest in perturbative approaches that go beyond the standard perturbative expansion [6, 7] by including partial resummations of higher-order terms. A variety of schemes have been developed, in both the Eulerian-space framework [8 14] and the Lagrangian-space framework [15 17]. However, most of these approaches are based on the single-stream approximation and neglect shell-crossing effects (a few exceptions are Refs. [17-19]).

To compare these theoretical predictions with observations, it is important to understand their range of validity. The impact of shell crossing onto the matter power spectrum has already been investigated in [20] and [21], using two variants of a phenomenological halo model or the Zel'dovich dynamics [22], and in [23] by estimating the generation of vorticity and velocity dispersion. In this paper, we investigate in more details the convergence of several perturbative expansions and the quantitative impact of nonperturbative effects due to shell crossing. Moreover, we estimate the sensitivity of the predicted power spectrum to the uncertainty of phenomenological parameters (the concentration of the halo density profile, the halo mass function) that must be taken from numerical simulations. This also gives an estimate of the accuracy of the power spectra obtained from these simulations.

To this order, we use the simple analytical model developed in [17], which combines one-loop standard perturbation theory with a halo model within a Lagrangianspace framework. This provides a good approximation to the nonlinear power spectrum on a broad range of scales and redshifts while being based on a physical modeling. Therefore, we can expect that it provides a good quantitative basis for such a study. For this article, the advantage of using such a toy model rather than numerical simulations is that we can easily separate perturbative from nonperturbative contributions, as well as the impact of different halo parameters. In contrast, numerical simulations include at once all these effects and this can lead to misleading comparisons with analytical approaches that neglect some of them.

This paper is organized as follows. In Sec. III we first recall the model for the power spectrum obtained in [17] that is the basis of our study. Then, in Sec. III we investigate the rate of convergence of some simple expansion schemes, both within an Eulerian-space and a Lagrangian-space framework. Next, in Sec. IV we estimate the importance of nonperturbative contributions, as a function of scale and redshift, and we consider the impact of the limited accuracy of phenomenological halo 
parameters. We conclude in Sec. V

\section{TOY MODEL FOR THE FULLY NONLINEAR MATTER POWER SPECTRUM}

We briefly recall in this section the expression of the matter power spectrum obtained in [17] (see the Appendix for details). As in usual halo models [3], this model writes the nonlinear power spectrum as a sum of one-halo and two-halo terms,

$$
P(k)=P_{1 \mathrm{H}}(k)+P_{2 \mathrm{H}}(k) .
$$

The one-halo term reads as

$$
P_{1 \mathrm{H}}(k)=\int_{0}^{\infty} \frac{\mathrm{d} \nu}{\nu} f(\nu) \frac{M}{\bar{\rho}(2 \pi)^{3}}\left(\tilde{u}_{M}(k)-\tilde{W}\left(k q_{M}\right)\right)^{2},
$$

where $f(\nu)$ is the scaling function that determines the halo mass function, as $\mathrm{d} n=\bar{\rho} / M f(\nu) \mathrm{d} \nu / \nu$, with $\nu=$ $\delta_{c} / \sigma(M)$ and $\sigma(M)$ the rms linear density contrast at mass scale $M$. Here, $\tilde{u}_{M}(k)$ is the Fourier transform of the density profile of a halo of mass $M$ [we use the popular Navarro-Frenk-White (NFW) profile [24]], given by Eq. A14), while $\tilde{W}\left(k q_{M}\right)$ is the Fourier transform of the top hat of Lagrangian radius $q_{M}$ [it also ensures that $P_{1 \mathrm{H}}(k) \propto k^{4}$ at low $k$ in agreement with the conservation of mass and momentum [25]]. Using a Lagrangian-space framework, the two-halo term reads as

$$
\begin{gathered}
P_{2 \mathrm{H}}(k)=\int \frac{\mathrm{d} \mathbf{q}}{(2 \pi)^{3}} F_{2 \mathrm{H}}(q)\left\langle e^{\mathrm{i} \mathbf{k} \cdot \mathbf{x}}\right\rangle_{q}^{\text {vir }} \frac{1}{1+A_{1}} \\
\times e^{-\frac{1}{2} k^{2}\left(1-\mu^{2}\right) \sigma_{\perp}^{2}}\left\{e^{-\varphi\left(-\mathrm{i} k q \mu \sigma_{\kappa}^{2}\right) / \sigma_{\kappa}^{2}}+A_{1}\right. \\
\left.+\int_{0^{+}-\mathrm{i} \infty}^{0^{+}+\mathrm{i} \infty} \frac{\mathrm{d} y}{2 \pi \mathrm{i}} e^{-\varphi(y) / \sigma_{\kappa}^{2}}\left(\frac{1}{y}-\frac{1}{y+\mathrm{i} k q \mu \sigma_{\kappa}^{2}}\right)\right\},
\end{gathered}
$$

where we integrate over the Lagrangian-space separation $\mathbf{q} \equiv \mathbf{q}_{2}-\mathbf{q}_{1}$ of particle pairs and $\mu=\mathbf{k} \cdot \mathbf{q} /(k q)$. Defining the displacement field $\Psi_{i}=\mathbf{x}_{i}-\mathbf{q}_{i}$, the longitudinal and transverse variances (with respect to the direction q) of the linear relative displacements are $\sigma_{\|}^{2}=$ $\left\langle\left(\Psi_{2 \| L}-\Psi_{1 \| L}\right)^{2}\right\rangle$ and $\sigma_{\perp}^{2}=\left\langle\left(\Psi_{2 \perp L}-\Psi_{1 \perp L}\right)^{2}\right\rangle$ (along any given transverse direction). They are given by Eqs. (A1)(A2). The dimensionless longitudinal relative displacement is denoted as $\kappa=x_{\|} / q=1+\Psi_{\|} / q$ and $\sigma_{\kappa}=\sigma_{\|} / q$. As explained in the Appendix, to derive Eq.(3), we approximated the transverse displacement as Gaussian (as in Lagrangian linear theory) whereas the longitudinal displacement is non-Gaussian, defined at the perturbative level by its cumulant generating function $\varphi(y)$,

$$
\varphi(y)=-\sum_{n=1}^{\infty} \frac{S_{n}^{\kappa}}{n !}(-y)^{n}, \quad S_{n}^{\kappa}=\frac{\left\langle\kappa^{n}\right\rangle_{c}}{\sigma_{\kappa}^{2(n-1)}},
$$

with the behavior at the origin $\left(S_{1}^{\kappa}=S_{2}^{\kappa}=1\right)$

$$
y \rightarrow 0: \quad \varphi(y)=y-\frac{y^{2}}{2}+S_{3}^{\kappa} \frac{y^{3}}{6}+\ldots
$$

This ensures that the underlying probability distribution function $\mathcal{P}_{\varphi}(\kappa)$, given by Eq. A8 , is normalized to unity and obeys the constraint $\langle\Psi\rangle=0$, and an adequate choice of the resummed function $\varphi$ also ensures that $\mathcal{P}_{\varphi}(\kappa)$ is everywhere positive. Finally, the factors $F_{2 \mathrm{H}},\left\langle e^{\mathrm{ik} \cdot \mathbf{x}}\right\rangle_{q}^{\mathrm{vir}}, A_{1}$, and the last integral over $y$, are nonperturbative shellcrossing contributions associated with pancake and halo formation (see 17] for details).

The nonlinear power spectrum (11) combines perturbation theory with a halo model. In particular, we showed in Ref. [17] that this power spectrum is exact up to second order $P_{L}^{2}$ if we use for the skewness $S_{3}^{\kappa}$ the expression (A7). Then, using for the resummed function $\varphi(y)$ the ansatz (A5)- , we checked that we obtained a good agreement with numerical simulations up to $k \sim 10 h \mathrm{Mpc}^{-1}$. Then, we can use the power spectrum (11) as a toy model to investigate the rate of convergence of various perturbative expansions to the resummed perturbative power or to estimate the impact of nonperturbative contributions and of halo parameters.

\section{CONVERGENCE OF SOME PERTURBATIVE EXPANSIONS}

\section{A. Eulerian-space expansions}

Throughout this paper, by "perturbative" we refer to quantities that can be expanded over integer powers of the linear power spectrum $P_{L}$, as in the standard perturbation theory [7]. Then, the perturbative part of the matter density power spectrum (11) writes as (see the Appendix and Ref.[17])

$$
P_{\text {pert. }}(k)=\int \frac{\mathrm{d} \mathbf{q}}{(2 \pi)^{3}} e^{-\varphi\left(-\mathrm{i} k q \mu \sigma_{\kappa}^{2}\right) / \sigma_{\kappa}^{2}} e^{-\frac{1}{2} k^{2}\left(1-\mu^{2}\right) \sigma_{\perp}^{2}} .
$$

It is also the perturbative part of the two-halo component (3), as the one-halo component (2) of the form $e^{-1 / \sigma^{2}}$ is nonperturbative. It is convenient to define the function $\psi(y)$, which describes the deviation from the Gaussian, by

$$
\psi(y) \equiv \varphi(y)-y+\frac{y^{2}}{2}=S_{3}^{\kappa} \frac{y^{3}}{6}+\ldots
$$

and the power spectrum (6) writes as

$$
\begin{aligned}
P_{\text {pert. }}(k)= & \int \frac{\mathrm{d} \mathbf{q}}{(2 \pi)^{3}} e^{\mathrm{i} k q \mu-\frac{1}{2} k^{2}\left[\mu^{2} \sigma_{\|}^{2}+\left(1-\mu^{2}\right) \sigma_{\perp}^{2}\right]} \\
& \times e^{-\psi\left(-\mathrm{i} k q \mu \sigma_{\kappa}^{2}\right) / \sigma_{\kappa}^{2}}
\end{aligned}
$$

Thus, setting $\psi=0$ in Eq. (8) gives back the Zel'dovich power spectrum 22, 26, 27], which is only exact up to linear order over $P_{L}$, whereas keeping $\psi \neq 0$ with Eq. A7 
gives a power spectrum that is exact up to second order $P_{L}^{2}$ and also generates approximate higher-order contributions 17.

In this framework, the functions $\varphi(y)$ and $\psi(y)$ depend on the scale $q$, but they do not depend on redshift nor on the amplitude of the linear power spectrum $P_{L}$. Therefore, the "standard" perturbative expansion over powers of $P_{L}$ of the power spectrum (11) can be recovered by expanding Eq. (88) over powers of $P_{L}$, that is, over powers of the linear displacement variances $\sigma_{\|}^{2}, \sigma_{\perp}^{2}$, and $\sigma_{\kappa}^{2}$. We denote this "standard perturbation theory" expansion as

$$
P_{\text {pert. }}(k)=\sum_{n=1}^{\infty} P_{\mathrm{SPT}}^{(n)}(k) \quad \text { with } \quad P_{\mathrm{SPT}}^{(n)} \propto\left(P_{L}\right)^{n},
$$

and from Eq.(8) each term reads as

$$
\begin{aligned}
& P_{\mathrm{SPT}}^{(n)}(k)=\int \frac{\mathrm{d} \mathbf{q}}{(2 \pi)^{3}} e^{\mathrm{i} k q \mu} \\
& \quad \times\left\lfloor e^{-\frac{1}{2} k^{2}\left[\mu^{2} \sigma_{\|}^{2}+\left(1-\mu^{2}\right) \sigma_{\perp}^{2}\right]-\psi\left(-\mathrm{i} k q \mu \sigma_{\kappa}^{2}\right) / \sigma_{\kappa}^{2}}\right\rfloor_{\left(P_{L}\right)^{n}},
\end{aligned}
$$

where $\lfloor. .\rfloor_{\left(P_{L}\right)^{n}}$ denotes the term of order $\left(P_{L}\right)^{n}$ of the expression between the two delimiters. Although the explicit expression (10) derives from the Lagrangian-space formulation (6) within our framework, the standard expansion of the form (9) is usually computed from a Eulerian-space approach. Being uniquely defined as the expansion over powers of $P_{L}$, the method of computation does not matter and no trace of the Lagrangian-space framework remains in this expansion, which can be fully defined within a Eulerian-space approach.

Because of the approximations involved in the model (6), this perturbative expansion is only exact up to second order $P_{L}^{2}$. However, we can expect its main features to be correct as the power spectrum built in Ref.[17] has been shown to provide a good quantitative match to numerical simulations and it is based on a realistic physical modeling [e.g., the probability distribution function $\mathcal{P}(\kappa)$ of relative displacements that underlies Eq.(6) is well behaved].

We show the first seven partial series of the expansion (9) in Fig. 11 We recover the well-known behavior of the standard perturbation theory [8, 21], which has already been exactly computed up to two-loop order, or up to very high order for the simpler Zel'dovich dynamics. As seen in the upper panel, the amplitude of higher orders grows increasingly fast at high $k$, so that the series (9) is badly behaved and cannot be used to compute the realspace correlation function because of the divergent high- $k$ tails. However, on quasilinear scales, $k \lesssim 0.4 h \mathrm{Mpc}^{-1}$ at $z=0.35$, the series seems to converge, at least up to order $N=7$. Nevertheless, the convergence is not very regular, as the series truncated at orders $N=2,4$, or 6 , shows a stronger deviation from the full perturbative power (8) than the previous orders $N=1,3$, or 5 (except on the very large scales). This faster convergence of odd-order partial series is even more clearly seen in the lower panel. This is due to the change of signs of the fast growing contributions $P_{\mathrm{SPT}}^{(n)}$. The nonlinear power spectrum (in the
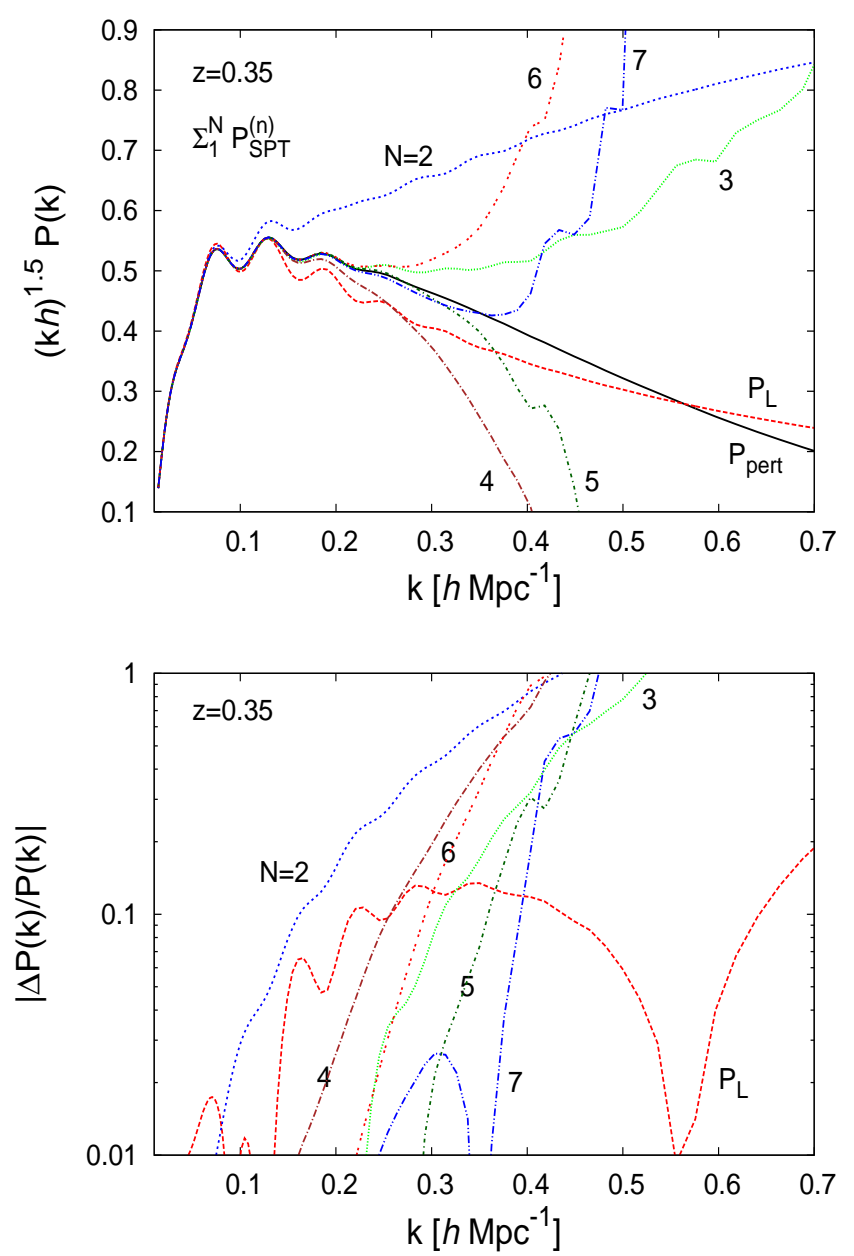

FIG. 1: Upper panel: "standard" perturbative expansion over powers of $P_{L}$ of the power spectrum (6), as in Eq. (9). We show the partial series truncated at order $N=1,2, . ., 7$ (the case $N=1$ is simply the linear power spectrum $P_{L}$ ), as well as the resummed perturbative power spectrum $P_{\text {pert. }}$, at redshift $z=$ 0.35. Lower panel: relative deviation between these partial series and the resummed perturbative power spectrum $P_{\text {pert. }}$ of Eq. (6).

convergence domain) arises from cancellations between the different terms $P^{(n)}$ and this explains why, for some values of $N$ going to order $N+1$ can worsen the result on scales that have not converged yet, because some required counterterms are included in the subsequent order $N+2$. In particular, while going to third order over $P_{L}$ (i.e., two-loop order in terms of the usual perturbative diagrams) significantly extends the range of validity of the prediction as compared with the linear or second-order approximations, a better approximation requires going to fifth order.

On the other hand, if we compare the partial series (9) with the full nonlinear power spectrum $P(k)$ measured in numerical simulations, or given by Eq.(11), we find that the second-order (i.e., one-loop) approximation 
fares best than all other truncations on a broad range of scale. This is because $k^{1.5} P_{1-\text { loop }}(k)$ happens to show a slow rise beyond quasilinear wave numbers that is similar to the growth shown by the nonlinear power spectrum. However, this is only a misleading coincidence: the "true" perturbative power spectrum $P_{\text {pert. }}$, of which $P_{1-\text { loop }}(k)$ is only a second-order approximation, actually shows a faster decrease at high $k$ and the growth of the nonlinear power spectrum $P(k)$ is due to nonperturbative effects that are not included in any perturbative scheme based on the single-stream approximation. This emphasizes the danger of comparing various perturbative approaches (or more general analytic models) with numerical simulations, which do not separate between the different contributions to the power spectrum (e.g., originating from perturbative and nonperturbative scales). Thus, a seemingly good agreement between a perturbative prediction and the full nonlinear power spectrum on transition scales is not necessarily meaningful. Because a non-negligible part of the power comes from effects that are not included in the model, a good match is likely to be a coincidence rather than the result of a very realistic and accurate modeling, and it may even become a problem as one tries to improve the model by adding these other effects.

As advocated in [8, 9], it is possible to reorganize the standard perturbation theory by factoring out a Gaussian damping term $e^{-k^{2} \sigma_{v}^{2}}$, where $\sigma_{v}^{2}=\left\langle\left|\Psi_{i}\right|^{2}\right\rangle / 3$ is the variance of the linear one-point displacement along one dimension. We denote this expansion as

$$
P_{\text {pert. }}(k)=e^{-k^{2} \sigma_{v}^{2}} \sum_{n=1}^{\infty} P_{\sigma_{v}}^{(n)}(k) \quad \text { with } \quad P_{\sigma_{v}}^{(n)} \propto\left(P_{L}\right)^{n}
$$

and from Eq.(8) each term reads as

$$
\begin{aligned}
& P_{\sigma_{v}}^{(n)}(k)=\int \frac{\mathrm{d} \mathbf{q}}{(2 \pi)^{3}} e^{\mathrm{i} k q \mu} \\
& \quad \times\left\lfloor e^{-\frac{1}{2} k^{2}\left[\mu^{2} \sigma_{\|}^{2}+\left(1-\mu^{2}\right) \sigma_{\perp}^{2}-2 \sigma_{v}^{2}\right]-\psi\left(-\mathrm{i} k q \mu \sigma_{\kappa}^{2}\right) / \sigma_{\kappa}^{2}}\right\rfloor_{\left(P_{L}\right)^{n}} .
\end{aligned}
$$

The prefactor $e^{-k^{2} \sigma_{v}^{2}}$ in Eq. (11) arises from the largedistance limit of the Gaussian term in Eq. (8). Indeed, at large separation length, $q \rightarrow \infty$, the two particles become uncorrelated and $\sigma_{\|}^{2}$ and $\sigma_{\perp}^{2}$ converge to $2 \sigma_{v}^{2}$. (This also means that for large $q$ the Gaussian term in Eq.(12) goes to zero, which simplifies the numerical computation.) Again, although the explicit expression (12) derives from a Lagrangian-space formulation, the expansion (11) is usually computed from a Eulerian-space approach and does not require introducing a Lagrangianspace framework.

The two expansions (9) and (11) can be derived from each other for any truncation order $N$. For instance, from the definitions (9) and (11) we obtain at once

$$
P_{\mathrm{SPT}}^{(n)}(k)=\sum_{p=0}^{n-1} \frac{\left(-k^{2} \sigma_{v}^{2}\right)^{p}}{p !} P_{\sigma_{v}}^{(n-p)}(k) .
$$
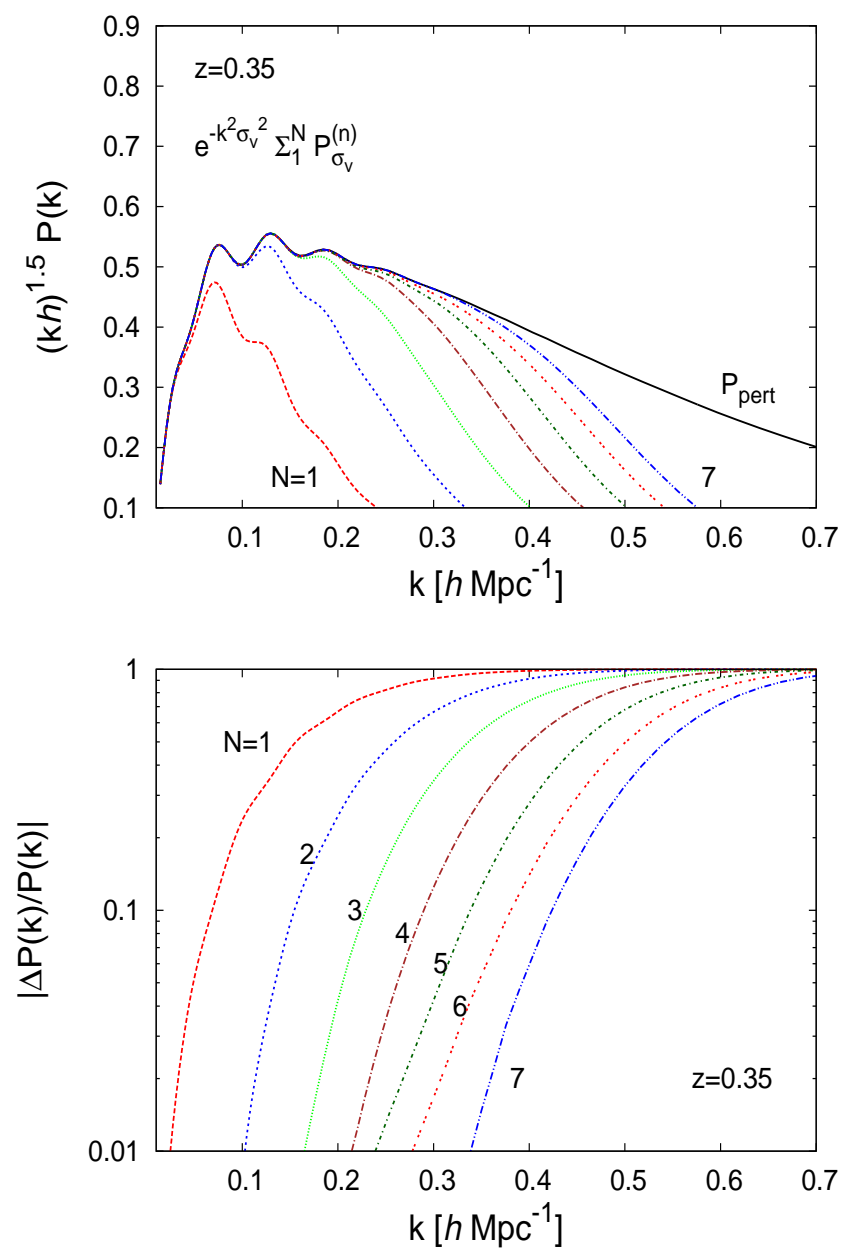

FIG. 2: Upper panel: reorganized perturbative expansion (11) of the power spectrum (6), with the Gaussian prefactor $e^{-k^{2} \sigma_{v}^{2}}$. We show the partial series truncated at order $N=1,2, . ., 7$, as well as the resummed perturbative power spectrum $P_{\text {pert. }}$ Lower panel: relative deviation between these partial series and the resummed perturbative power spectrum $P_{\text {pert. of Eq. (6). }}$.

(This is in fact how we computed the standard high-order terms $P_{\mathrm{SPT}}^{(n)}$ because the terms $P_{\sigma_{v}}^{(n)}$ are better behaved.)

We show the first seven partial series of the expansion (12) in Fig. 2. We recover the well-known property 8, 21] that this reorganized expansion is much better behaved than the standard expansion (9). This is partly artificial as this is mostly due to the too strong Gaussian cutoff $e^{-k^{2} \sigma_{v}^{2}}$. As explained for instance in [27, 28], this damping only occurs for different-time propagators or power spectra and vanishes for equal-time statistics, which show a smoother power-law decline at high $k$ (as for the Zel'dovich power spectrum 26, 27, 29]|). Then, the series in Eq.(11) must compensate for this too strong cutoff and behave as $e^{k^{2} \sigma_{v}^{2}}$, up to power-law corrections, which gives contributions of the form $e^{-k^{2} \sigma_{v}^{2}} P_{\sigma_{v}}^{(n)}(k) \sim$ 
$e^{-k^{2} \sigma_{v}^{2}}\left(k \sigma_{v}\right)^{2 n} / n$ ! that are positive and well ordered, with a sharp peak around $k_{n} \sim \sqrt{n} / \sigma_{v}$. Nevertheless, this reorganization of the perturbative expansion provides a very regular convergence to the perturbative power spectrum (6), at least up to order $N=7$, The comparison of Fig. 2 with the standard expansion displayed in Fig. 1 shows that even-order series $(N=2,4,6)$ are significantly improved while odd-order series $(N=1,3,5,7)$ fare somewhat worse. However, the well-ordered convergence of the expansion (11) (at least on these scales, as the radius of convergence of the perturbative series is not necessarily infinite) makes it superior to the standard expansion. Another key advantage of the series (11) is that the high- $k$ tail is no longer divergent. This means that we can now compute the Fourier transform of the power spectrum (11), which gives a perturbative expansion of the two-point correlation $\xi(x)$.

We show in Fig. 3 the correlation functions $\xi_{\sigma_{v}}^{(\leq N)}$ obtained from the partial series (11) truncated at order $N$. We compare these results with the correlation function $\xi_{\text {pert. }}$ defined by the resummed perturbative power spectrum of Eq. (6). We focus on BAO scales because small nonlinear scales are beyond the reach of this approach. As compared with linear theory, we can see that the simple multiplication of the linear power spectrum by the Gaussian damping $e^{-k^{2} \sigma_{v}^{2}}$ already provides a very significant improvement, as the deviation from the resummed correlation $\xi_{\text {pert. }}$ decreases from $30 \%$ to $4 \%$, at redshift $z=0.35$. The second (one-loop) order already gives a better than percent accuracy. (The rise of the curves in the lower panel at $x \sim 130 h^{-1} \mathrm{Mpc}$ is due to the change of sign and crossing through zero of $\xi_{\text {pert., }}$ which amplifies relative deviations.) Thus, perturbative expansions converge very fast for the real-space BAO peak, provided the high- $k$ tail of their power spectrum is well behaved. This explains why most perturbative resummation schemes manage to give accurate predictions for the $\mathrm{BAO}$ correlation function.

The comparison between Figs. 2 and 3 shows that the convergence is much faster for the correlation function than for the power spectrum. This means that the realspace BAO peak is a more robust probe of cosmology than the oscillations of the power spectrum. This is because by looking at the BAO peak we focus on a fixed scale, far in the quasilinear regime, whereas by looking at oscillations in $P(k)$ we must consider a broad range of wave numbers, where different orders contribute, including nonperturbative effects that are not shown in Fig. 2 More generally, the power spectrum and the correlation function are not identical probes for practical purposes, because the Fourier transform mixes all scales, to some degree, and we can never observe all scales nor make accurate predictions for all scales.
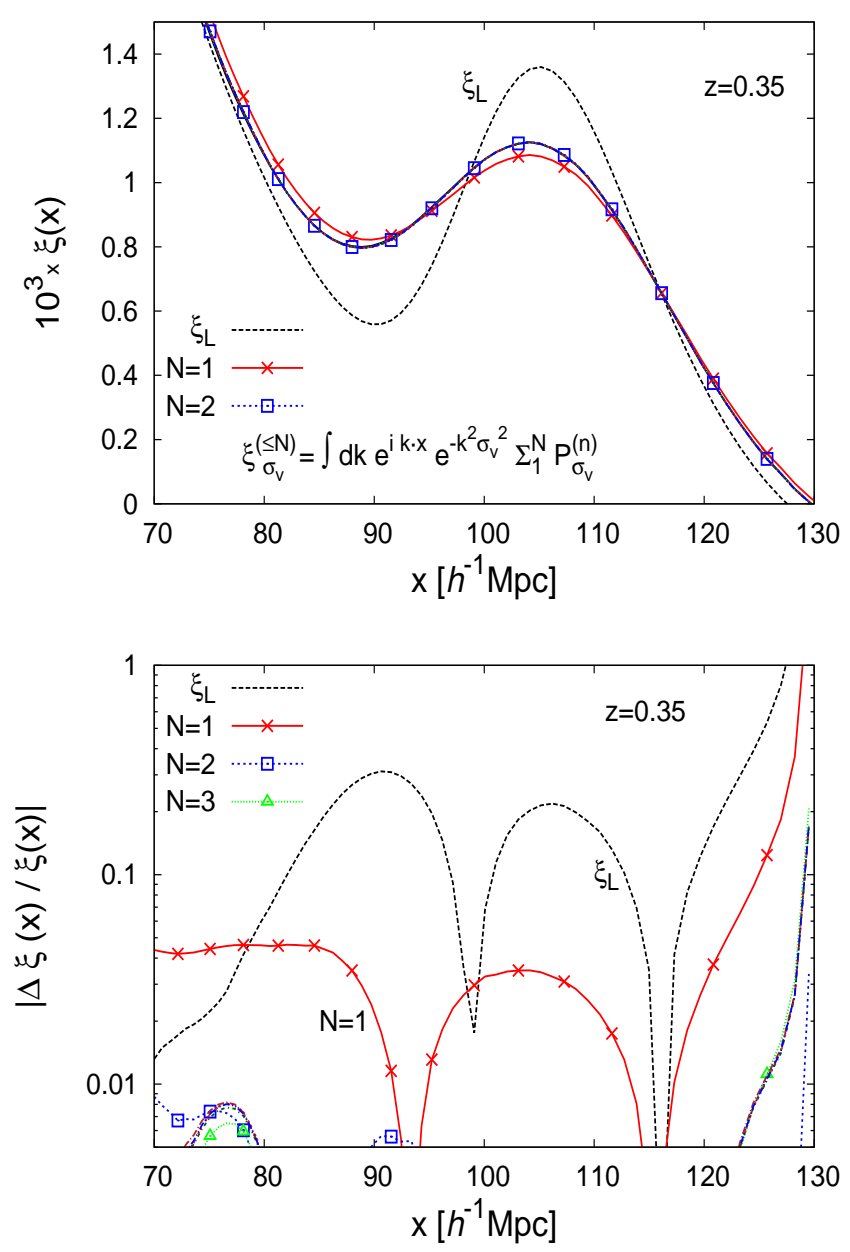

FIG. 3: Upper panel: two-point correlation functions $\xi_{\sigma_{v}}^{(\leq N)}(x)$ defined by the partial series for the power spectrum shown in Fig. 2 and given by Eq.(11). We show the partial series truncated at order $N=1,2, . ., 7$, as well as the resummed correlation $\xi_{\text {pert. }}$ and the linear correlation $\xi_{L}$. The curves for $N \geq 2$ and $\xi_{\text {pert. }}$ cannot be distinguished. Lower panel: relative deviation between these perturbative expansions $\xi_{\sigma v}^{(\leq N)}$ and the resummed correlation $\xi_{\text {pert. }}$.

\section{B. Lagrangian-space expansions}

We have described in Sec. ЩIA the two simplest perturbative expansions of the density power spectrum. Being defined as the truncation at order $N$ of expansions over powers of $P_{L}$, they can be computed by any perturbative method, using either a Eulerian or Lagrangian framework. In practice, they are computed using the standard Eulerian perturbation theory [7], which is the simplest approach and directly provides these partial series. However, because our model (6) is based on a Lagrangian-space framework, it also allows us to investigate the standard Lagrangian perturbation theory. In this approach, instead of looking for a perturbative ex- 
pansion of the density and velocity fields, which gives in turn the density and velocity power spectra, one looks for a perturbative expansion of the displacement field, $\Psi_{i}=\mathbf{x}_{i}-\mathbf{q}_{i}$. This gives in turn the density power spectrum through the relation

$$
\begin{aligned}
P(k) & =\int \frac{\mathrm{d} \mathbf{q}}{(2 \pi)^{3}}\left\langle e^{\mathrm{i} \mathbf{k} \cdot \mathbf{x}}\right\rangle \\
& =\int \frac{\mathrm{d} \mathbf{q}}{(2 \pi)^{3}} \exp \left[\sum_{n=1}^{\infty} \frac{\left\langle(\mathrm{ik} \cdot \mathbf{x})^{n}\right\rangle_{c}}{n !}\right],
\end{aligned}
$$

where again $\mathbf{q}=\mathbf{q}_{2}-\mathbf{q}_{1}$ and $\mathbf{x}=\mathbf{x}_{2}-\mathbf{x}_{1}$ are the Lagrangian and Eulerian pair separations (and we discarded a Dirac factor). If we expand the exponential over powers of $P_{L}$ we recover the standard Eulerian perturbation theory, as in Sec. IIIA. but by keeping some terms in the exponential we obtain alternative approximations, which can be seen as partial resummations of the standard Eulerian perturbation theory.

If we truncate the cumulant series in the exponential in Eq. 15) at order $P_{L}$, which corresponds to the linear displacement field, we recover the Zel'dovich power spectrum,

$$
P_{\mathrm{Z}}(k)=\int \frac{\mathrm{d} \mathbf{q}}{(2 \pi)^{3}} e^{\mathrm{i} k q \mu-\frac{1}{2} k^{2}\left[\mu^{2} \sigma_{\|}^{2}+\left(1-\mu^{2}\right) \sigma_{\perp}^{2}\right]},
$$

which coincides with Eq. (8) where we set $\psi=0$. This is only exact up to linear order over $P_{L}$. Then, to go beyond the Zel'dovich approximation, we usually compute the displacement field up to some finite order over the amplitude of the initial fluctuations $\delta_{L}$, substitute into Eq. (14) and expand the exponential over terms that are cubic or higher order over $\delta_{L}$. This provides a Gaussian expression over $\delta_{L}$, with polynomial prefactors, that can be explicitly computed. Within our framework (6), this simply corresponds to expanding the last term in Eq. (8) over powers of $P_{L}$. This gives the perturbative expansion

$$
P_{\text {pert. }}(k)=\sum_{n=1}^{\infty} P_{\mathrm{Z}}^{(n)}(k) \quad \text { with } \quad P_{\mathrm{Z}}^{(n)} \sim \mathcal{O}\left[\left(P_{L}\right)^{n}\right],
$$

and

$$
\begin{aligned}
n \geq 2: \quad P_{\mathbf{Z}}^{(n)}(k)= & \int \frac{\mathrm{d} \mathbf{q}}{(2 \pi)^{3}} e^{\mathrm{i} k q \mu-\frac{1}{2} k^{2}\left[\mu^{2} \sigma_{\|}^{2}+\left(1-\mu^{2}\right) \sigma_{\perp}^{2}\right]} \\
& \times\left\lfloor e^{-\psi\left(-\mathrm{i} k q \mu \sigma_{\kappa}^{2}\right) / \sigma_{\kappa}^{2}}\right\rfloor_{\left(P_{L}\right)^{n}}
\end{aligned}
$$

and we define $P_{\mathrm{Z}}^{(1)}=P_{\mathrm{Z}}$. Thus, each term $P_{\mathrm{Z}}^{(n)}$ scales as $\left(P_{L}\right)^{n}$ for $P_{L} \rightarrow 0$ but it also contains contributions at all higher orders. The first order $n=1$ is the Zel'dovich power spectrum. The second order $n=2$ involves the skewness $S_{3}^{\kappa}$.

We show the first six partial series of the expansion (17) in Fig. 目 As compared with the Eulerian expansions, we can see that the first few partial series converge much faster and on a broader range of scales. Moreover, the accuracy improves with the order from $N=1$ to $N=3$, but
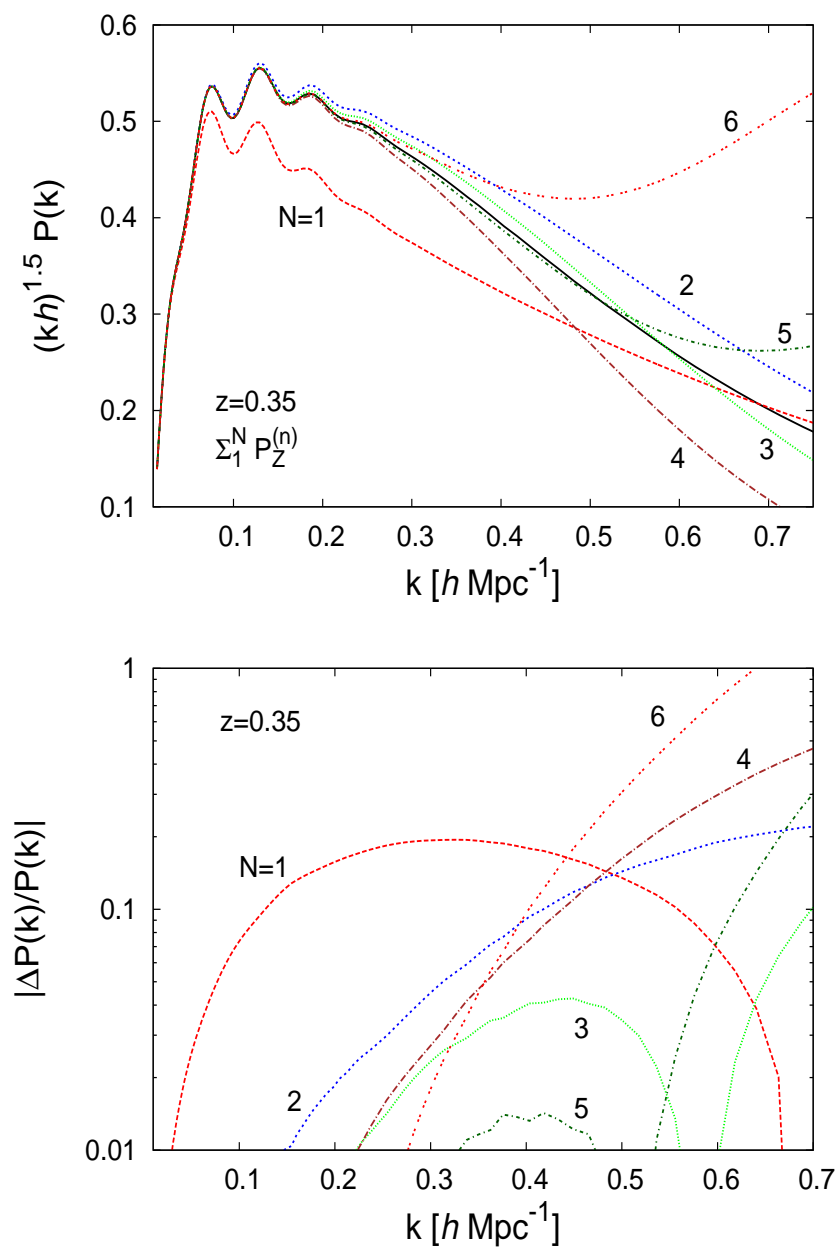

FIG. 4: Upper panel: Lagrangian-space perturbative expansion (17) of the power spectrum (8). We show the partial series truncated at order $N=1,2, . ., 6$, as well as the resummed perturbative power spectrum $P_{\text {pert. }}$ Lower panel: relative deviation between these partial series and the resummed perturbative power spectrum $P_{\text {pert. }}$ of Eq. (8).

the orders $N=4$ and $N=6$ are somewhat worse than $N=3$ and $N=5$. This suggests that this Lagrangianspace perturbative expansion has a better start because the Zel'dovich approximation is a good starting point (which is sometimes used for instance to initialize numerical simulations), as it corresponds to a physical matter distribution that is realistic on large scales and well defined beyond shell crossing. In particular, the high- $k$ tail remains well behaved until $N \leq 4$. However, for high orders, $N=5,6$, the series does not seem to converge very well and the high- $k$ tail starts diverging. Therefore, the Lagrangian-space expansion (17) shares some features with both Eulerian-space expansions (9) and (11). The first few orders show an ordered systematic convergence, as for (11), but at higher orders the convergence becomes irregular, as in (9), and may even break down 
at high $k$. Nevertheless, this Lagrangian-space expansion appears superior to both Eulerian-space expansions in the sense that for a given low order (e.g., $N=3$ or 5 ), the accuracy is significantly better and has a broader range of validity. However, it seems that it should not be pushed too far. The bad convergence at high $k$ and large orders may be due to a finite radius of convergence of perturbative expansions of the power spectrum (6), associated with singularities of the function $\varphi(y)$ in the complex plane at a finite distance from the origin. Then, the Eulerian-space expansions (9) and (11) are expected to show the same problems as we push them to higher orders and higher $k$. On the other, this high-order behavior may not generalize to the exact gravitational dynamics, where the conditions of convergence of perturbative expansions are not known.

We show the correlation functions $\xi_{Z}^{(\leq N)}$ obtained from the partial series (17) in Fig. 5 We again focus on BAO scales, for the orders $N=1,2$, and 3 . As for the reorganized Eulerian-space expansion (11) shown in Fig. 3, the first term $N=1$ (which is the Zel'dovich approximation here) already improves the accuracy from $30 \%$ to $4 \%$ at redshift $z=0.35$, as compared with linear theory. This reasonably good agreement of the Zel'dovich correlation function with numerical simulations on large scales was already noticed in [17, 30]. Orders $N=2$ and 3 provide a subpercent accuracy. The accuracy is only slightly better than for the expansion (11) at these orders.

An alternative to the approach associated with the expansion (18) is to again compute the displacement field up to some finite order over the initial fluctuations $\delta_{L}$, but then to substitute into Eq.(15) and keep a truncated cumulant series into the exponential. Within our framework (8), this simply corresponds to expanding the function $\psi$ in the exponential. This gives the sequence of approximations

$$
\begin{aligned}
& P_{\mathrm{ZE}}^{(\leq N)}(k)=\int \frac{\mathrm{d} \mathbf{q}}{(2 \pi)^{3}} e^{\mathrm{i} k q \mu-\frac{1}{2} k^{2}\left[\mu^{2} \sigma_{\|}^{2}+\left(1-\mu^{2}\right) \sigma_{\perp}^{2}\right]} \\
& \times e^{\left\lfloor-\psi\left(-\mathrm{i} k q \mu \sigma_{\kappa}^{2}\right) / \sigma_{\kappa}^{2}\right\rfloor \leq\left(P_{L}\right)^{N}},
\end{aligned}
$$

where $\lfloor. .\rfloor_{\leq\left(P_{L}\right)^{N}}$ denotes the truncation at order $N$ over $P_{L}$ of the expression between the two delimiters. The "E" in the subscript "ZE" recalls that we keep the terms in the exponential. The order $N=1$ again corresponds to the Zel'dovich power spectrum (16).

We show the first seven orders of the expansion (19) in Fig. 6. We obtain a behavior that is similar to the one

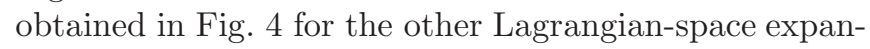
sion (17). We again find a fast convergence of the first few orders at $k \leq 0.5 \mathrm{hppc}^{-1}$ and signs of bad behavior and divergence at higher orders and higher $k$. The accuracy is typically of the same order as for the expansion (17). This means that on quasilinear scales there is not much difference between expanding the exponential or not. The high- $k$ tail is more sensitive to the details of the method but it also seems beyond the scope of these perturbative expansions.
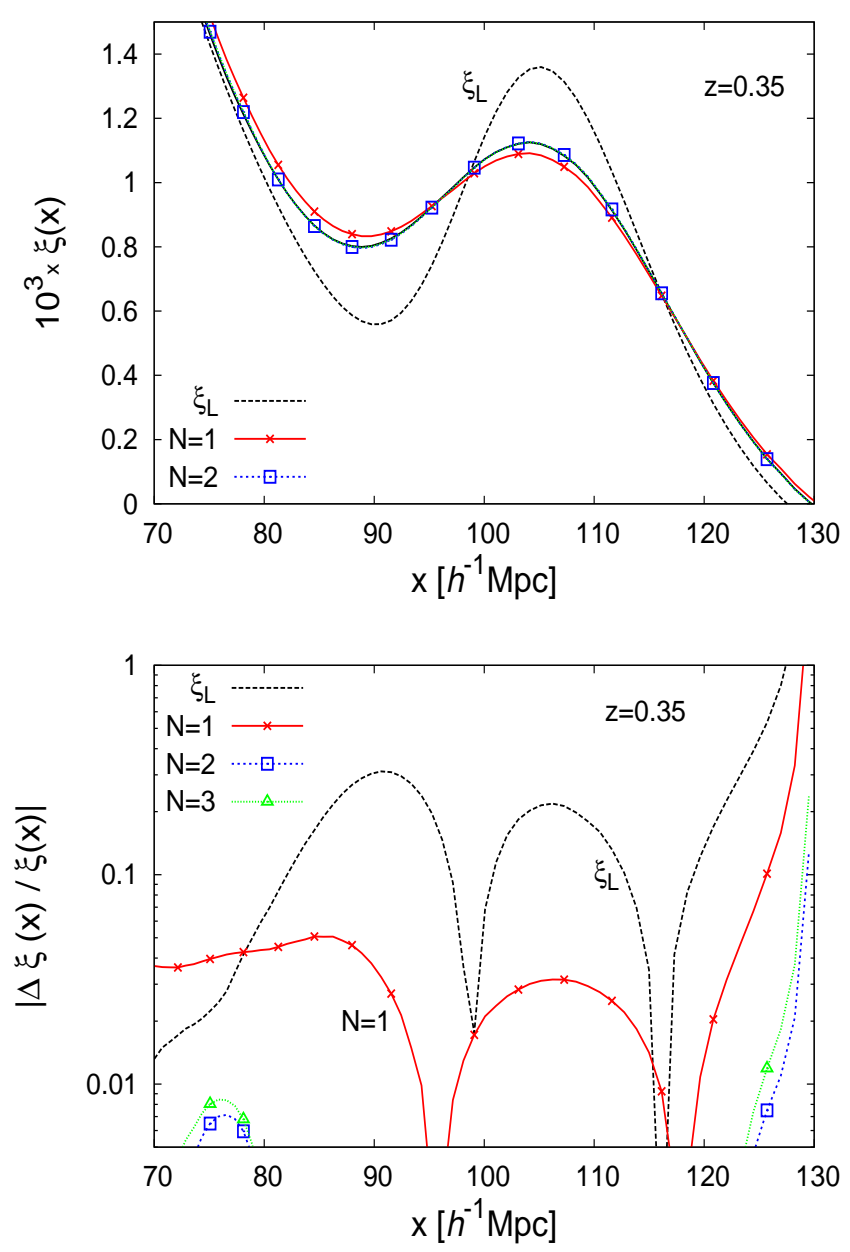

FIG. 5: Upper panel: two-point correlation functions $\xi_{\mathrm{Z}}^{(\leq N)}(x)$ defined by the partial series for the power spectrum shown in Fig. 4 and given by Eq. (17). We show the partial series truncated at order $N=1,2$, and 3 , as well as the resummed correlation $\xi_{\text {pert. }}$ and the linear correlation $\xi_{L}$. The curves for $N=2,3$, and $\xi_{\text {pert. }}$, cannot be distinguished. Lower panel: relative deviation between these perturbative expansions $\xi_{\sigma_{v}}^{(\leq N)}$ and the resummed correlation $\xi_{\text {pert. }}$

We also computed the two-point correlations $\xi_{\mathrm{ZE}}^{(\leq N)}$ for $N=1$ and 2 and found results that are similar to those in Fig. 5 .

Thus, we can conclude that for a fixed low order of truncation, Lagrangian-space approaches can have a broader range of validity than their Eulerian counterparts for the power spectrum and also provide good approximations to the two-point correlation on $\mathrm{BAO}$ scales. However, for the computation of the BAO peak of the two-point correlation, the simple reorganization (11) of standard Eulerian perturbation theory may be more efficient because of its greater simplicity. 

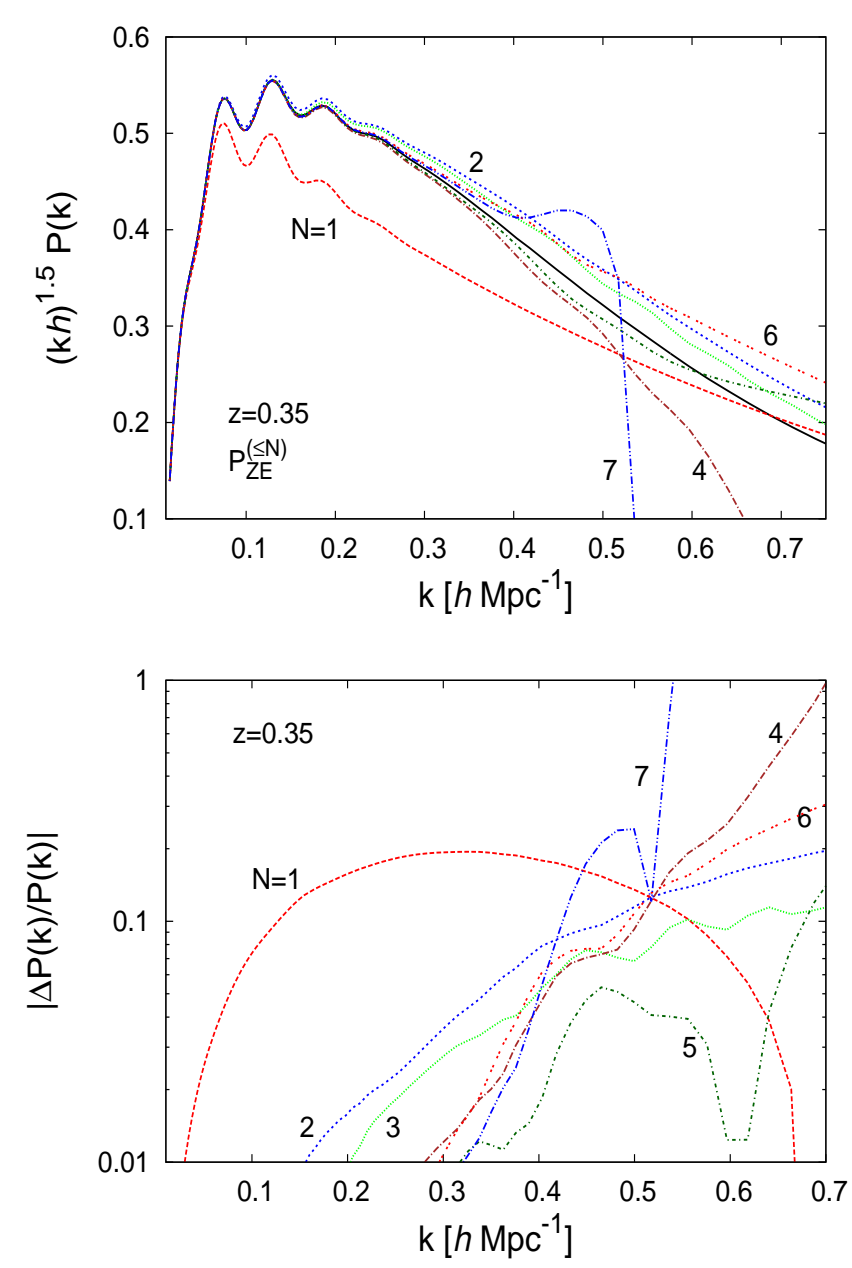

FIG. 6: Upper panel: Lagrangian-space perturbative expansion (19) of the power spectrum (8). We show the orders $N=1,2, . ., 7$, as well as the resummed perturbative power spectrum $P_{\text {pert. }}$ Lower panel: relative deviation between these partial series and the resummed perturbative power spectrum $P_{\text {pert. }}$ of Eq. (8).

\section{SCOPE OF PERTURBATIVE APPROACHES}

\section{A. Impact of nonperturbative contributions}

We have investigated in Sec. IIIthe convergence of Eulerian and Lagrangian perturbative expansions toward the resummed perturbative power spectrum (6). However, even if we find an efficient perturbative scheme, or manage to resum the perturbative expansion, this is not sufficient to provide the matter density power spectrum. Indeed, these perturbative expansions are restricted to the single-stream regime and do not include nonperturbative effects associated with shell crossings. (Even though the Lagrangian expansions of Sec. IIIB implicitly go beyond shell crossing, as in the Zel'dovich approximation, this analytic continuation is not exact and cannot be trusted in this regime.)

Our framework (11)-(3), based on the halo model, combines these perturbative single-stream contributions with nonperturbative contributions that are modeled in a more phenomenological fashion. However, because our model has been shown to provide a good agreement with numerical simulations for a variety of cosmologies [17] and it is based on a realistic modelization, it allows us to estimate the relative importance of these contributions. We can split the full nonlinear power spectrum into three components,

$$
P(k)=P_{\text {pert. }}(k)+P_{2 \mathrm{H}}^{\text {nonpert. }}(k)+P_{1 \mathrm{H}}(k) .
$$

The one-halo term $P_{1 \mathrm{H}}$ is the fully nonperturbative contribution given by Eq.(2) and we split the two-halo term given by Eq. (3) into its perturbative part $P_{\text {pert. }}$, given by Eq.(6), and its nonperturbative part $P_{2 \mathrm{H}}^{\text {nonpert. }}$, defined as

$$
P_{2 \mathrm{H}}^{\text {nonpert. }}(k) \equiv P_{2 \mathrm{H}}(k)-P_{\text {pert. }}(k) .
$$

Of course, the halo model itself is only an approximate and phenomenological description of the density field. Hence the splitting of nonperturbative contributions into the two terms $P_{2 \mathrm{H}}^{\text {nonpert. }}$ and $P_{1 \mathrm{H}}$ is not very well and uniquely defined, especially from a Eulerian point of view. However, from the Lagrangian point of view that led to the model (11)-(3), the distinction is easier to make (even though approximate) as pairs of particles belong either to the same or different halos and we can split their motion into small-scale virial motions and large-scale collective flows. Then, the term $P_{2 \mathrm{H}}^{\text {nonpert. }}$ is the contribution of small-scale multistreaming to the large-scale power spectrum. This transfer of power is due to the Fourier transform that defines the power spectrum, which mixes scales: finite-scale motions contribute to all wave numbers in Eq.(14). Within our framework (3), this arises from the uncorrelated small-scale virial motions of both particles in their respective halos [the factor $\left\langle\left. e^{\mathbf{i k} \cdot \mathbf{x}}\right|_{q} ^{\mathrm{vir}}\right.$ in Eq.(3)], the sticking of particles within pancakes (instead of escaping to infinity as in the Zel'dovich approximation) [the factors $A_{1}$ and the complex integral over $y$ in Eq.(33)], and the removal of particle pairs that belong to the same halo [the factor $F_{2 \mathrm{H}}$ in Eq.(3)] as we collect their contribution in a separate one-halo term. At a qualitative level, these factors are analogous to the viscous (sticking in pancakes) and pressure (uncorrelated small-scale virial motions) terms that have been added in some previous works [18, 19] to the hydrodynamical equations of motion to build perturbative expansions that can handle some shell-crossing effects. These effective approaches, which are based on a separation of scales, would provide another route to predict the "cosmic web" power spectrum or two-halo term (3) but cannot describe the fully nonlinear scales associated with the one-halo term (2).

We show in Fig. (7) the three components of Eq.(20). We can check that the perturbative component is dominant on large scales, the one-halo term on small scales, and 

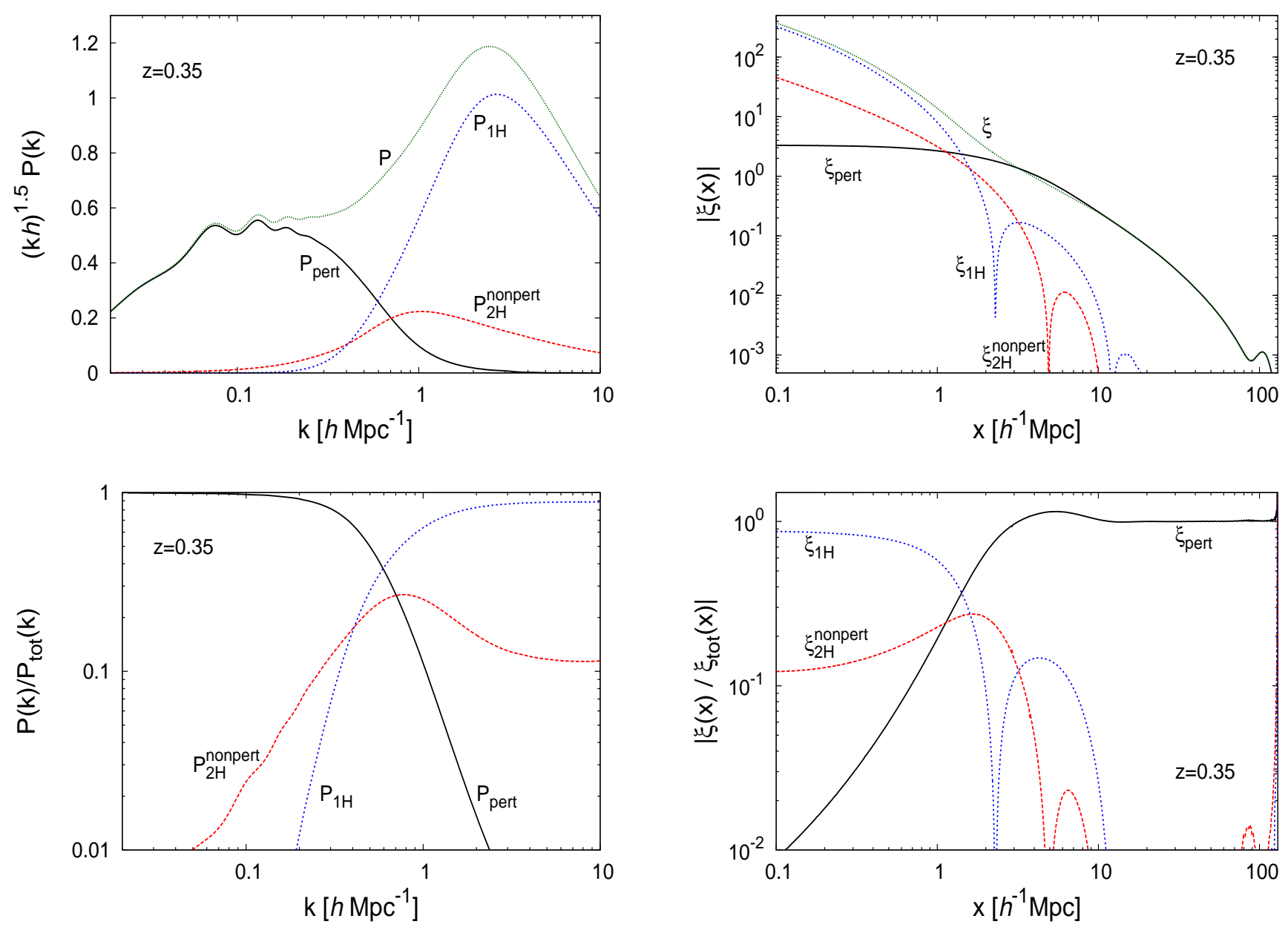

FIG. 7: Upper panel: perturbative and nonperturbative contributions to the full nonlinear density power spectrum, from Eq. (20), at $z=0.35$. Lower panel: relative importance of these contributions to the full power spectrum. $P_{\text {pert. }}(k)$ is obtained from Eq. (6), $P_{1 \mathrm{H}}(k)$ from Eq.(2), and $P_{2 \mathrm{H}}^{\text {nonpert. }}(k)$ from Eqs. (3) and (21).

the nonperturbative part of the two-halo term is mainly relevant on intermediate scales. As seen in the lower panel, the perturbative part $P_{\text {pert. }}$ shows a fast decrease at high $k$ in relative terms. This is because it typically decays faster than $k^{-3}$ as the Zel'dovich power spectrum, because intermediate-scale structures are erased as particles keep moving on. The one-halo term (2) shows a fast decrease at low $k$ because of its $k^{4}$ tail, ensured by the counterterm $\tilde{W}$ in Eq.(2), associated with mass and momentum conservation. The term $P_{2 \mathrm{H}}^{\text {nonpert. }}$, which combines small and large scales, shows a broader distribution but its precise shape is likely to depend on the details of our model. In any case, Fig. 7 shows that if we require a few percent accuracy we are sensitive to shell-crossing effects down to $k \sim 0.1 \mathrm{hpc}^{-1}$ at $z=0.35$. This agrees within a factor of about 2 with the results obtained in

FIG. 8: Upper panel: perturbative and nonperturbative contributions to the full nonlinear density correlation function, from Eq.22), at $z=0.35$. Lower panel: relative importance of these contributions to the full correlation function.

previous works that used simpler models 20, 21]. As expected, we first encounter the term $P_{2 \mathrm{H}}^{\text {nonpert. }}$, associated with the residual effect of small-scale multistreaming onto the large-scale power, and next the one-halo contribution.

We must note that Fig. 7 does not define by itself the limitation of semianalytical models. This only gives a lower bound to the range of wave numbers that can be described by semianalytical models, if we set all nonperturbative contributions to zero and use a Lagrangianbased regularization of perturbation theory. In practice, if we take into account nonperturbative contributions in an approximate fashion, as in Ref. [17] for instance or by including some additional pressure terms to the equations of motion, we can extend the range of validity of semianalytical models. In particular, they only need to be modeled up to $10 \%$ on scales where they do not contribute to more than $20 \%$ if we require a $2 \%$ accuracy. We discuss in more details these points in Secs. IVB 1 and IVB2 
below. However, Fig. 7 is useful as a warning to the limitations of perturbation theories and gives an estimate of the scale and accuracy where adding high-order contributions to the single-stream perturbative expansions is relevant.

The Fourier transform of Eq.(20) gives the decomposition of the two-point correlation function,

$$
\xi(x)=\xi_{\text {pert. }} .(x)+\xi_{2 \mathrm{H}}^{\text {nonpert. }}(x)+\xi_{1 \mathrm{H}}(x) .
$$

We show our results in Fig. 8, Again, the perturbative term dominates on large scales, the one-halo term on small scales, and the nonperturbative part of the twohalo term is mainly relevant on intermediate scales. The peaks at $x \simeq 130 h^{-1} \mathrm{Mpc}$ in the lower panel are due to the zero crossing of the two-point correlation, which makes the ratios diverge. The lower panel shows that nonperturbative contributions are negligible at $z=0.35$ on scales larger than $10 h^{-1} \mathrm{Mpc}$, even when we require an accuracy of $1 \%$. (This also agrees with Ref. [30] who noticed that the Zel'dovich correlation function is reasonably accurate down to $\sim 10 h^{-1} \mathrm{Mpc}$.) This shows that real-space statistics provide a robust and efficient probe of cosmology as they offer a clean separation between perturbative and nonperturbative contributions. This is important as the perturbative contributions can be computed from first principles by systematic expansion schemes, whereas nonperturbative contributions are necessarily more phenomenological and of limited accuracy. Moreover, as noticed in Sec. III, the perturbative expansions of the two-point correlation function show a fast convergence on large scales. These features can be understood from the physics at play, as nonlinearities arise from small-scale motions (rather than wave interactions) that take place in configuration space and do not redistribute matter on large scales. Then, one can expect a separation of scales to be more readily apparent in configuration space than in Fourier space, where the integral transform spreads the contributions to all wave numbers.

To study the evolution with redshift of these perturbative and nonperturbative contributions, we show in the upper panel of Fig. 9 the contour lines in the $(k, z)$-plane of the regions where $P_{2 \mathrm{H}}^{\text {nonpert. }}$ and $P_{1 \mathrm{H}}$ contribute to more than $2 \%$ or $10 \%$ of the full power spectrum. The lower panel shows the same contour lines for the two-point correlation in the $(x, z)$-plane. At higher redshift the region where the nonperturbative contributions are important is pushed toward higher wave numbers $k$ and smaller scales $x$. The contour lines associated with $P_{2 \mathrm{H}}^{\text {nonpert. }}$ and $P_{1 \mathrm{H}}$ are similar, as they follow the nonlinear scale. The lower panel shows that if we can model these nonperturbative up to $20 \%$ we can reach an accuracy of $2 \%$ for $\xi(x)$ down to $x \geq 10$ or $x \geq 0.5 h^{-1} \mathrm{Mpc}$ at redshifts $z=0$ or 3 (provided we have a good perturbative scheme, but this should not be the limiting factor). This is rather reassuring, as it ensures a broad range of scales for future wide surveys.
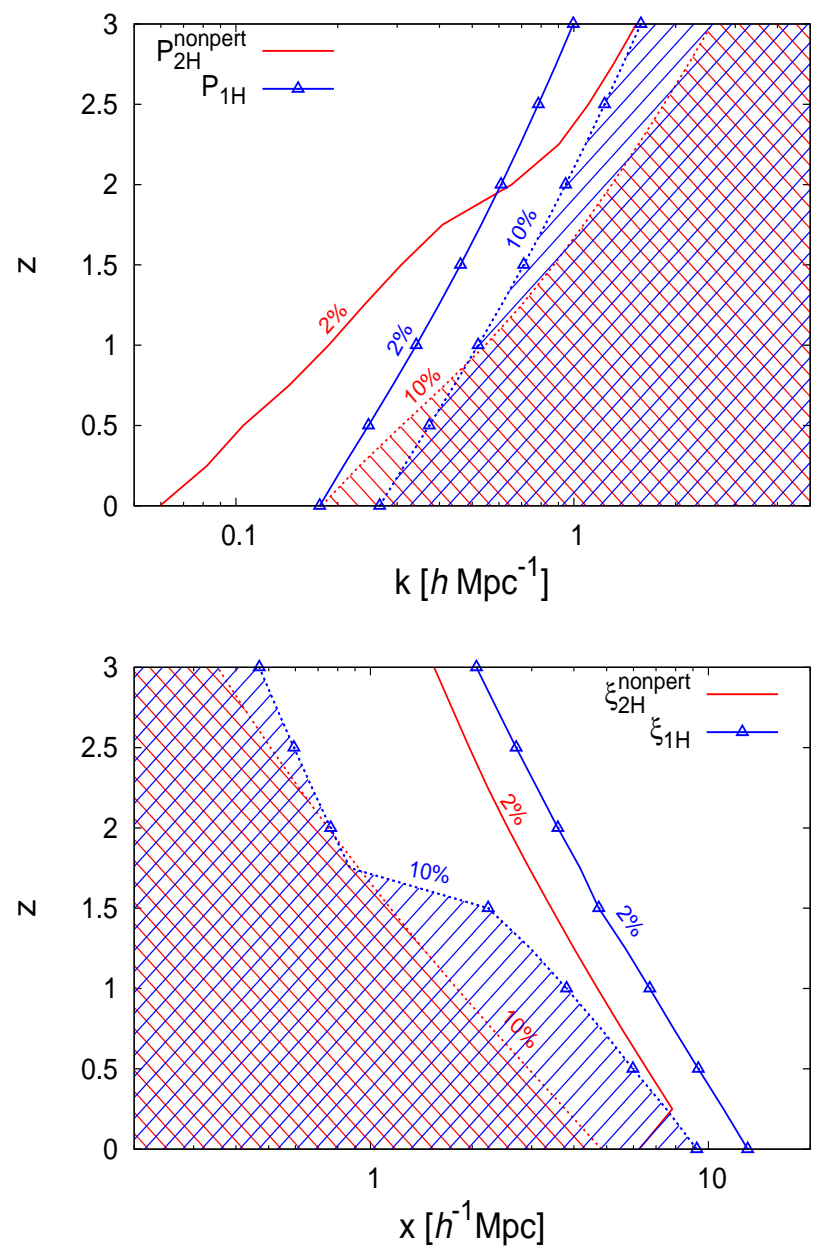

FIG. 9: Upper panel: contour lines in the $(k, z)$-plane of the regions where $P_{2 \mathrm{H}}^{\text {nonpert. }}$ or $P_{1 \mathrm{H}}$ (lines with triangle symbols) make more than $2 \%$ (solid lines) or $10 \%$ (dotted lines) of the full power spectrum. Lower panel: similar contour lines in the $(x, z)$-plane for the relative contributions of $\xi_{2 \mathrm{H}}^{\text {nonpert. }}$ and $\xi_{1 \mathrm{H}}$ to the full two-point correlation $\xi$.

\section{B. Impact of phenomenological parameters}

As noticed in Sec. IVA the study of the relative importance of nonperturbative contributions to the matter power spectrum or correlation function only gives a very conservative estimate of the scope of semianalytical models. Indeed, the latter can include such effects in a phenomenological manner, e.g. through a halo model, or through an implicit regularization of the perturbative scheme. Then, we can estimate the scope of semianalytical models by studying the sensitivity of their predictions to the value of these phenomenological parameters, which cannot be computed by systematic analytical approaches. Within our framework, this corresponds to investigating the sensitivity of our predictions on the halo-model parameters, associated with the halo mass 

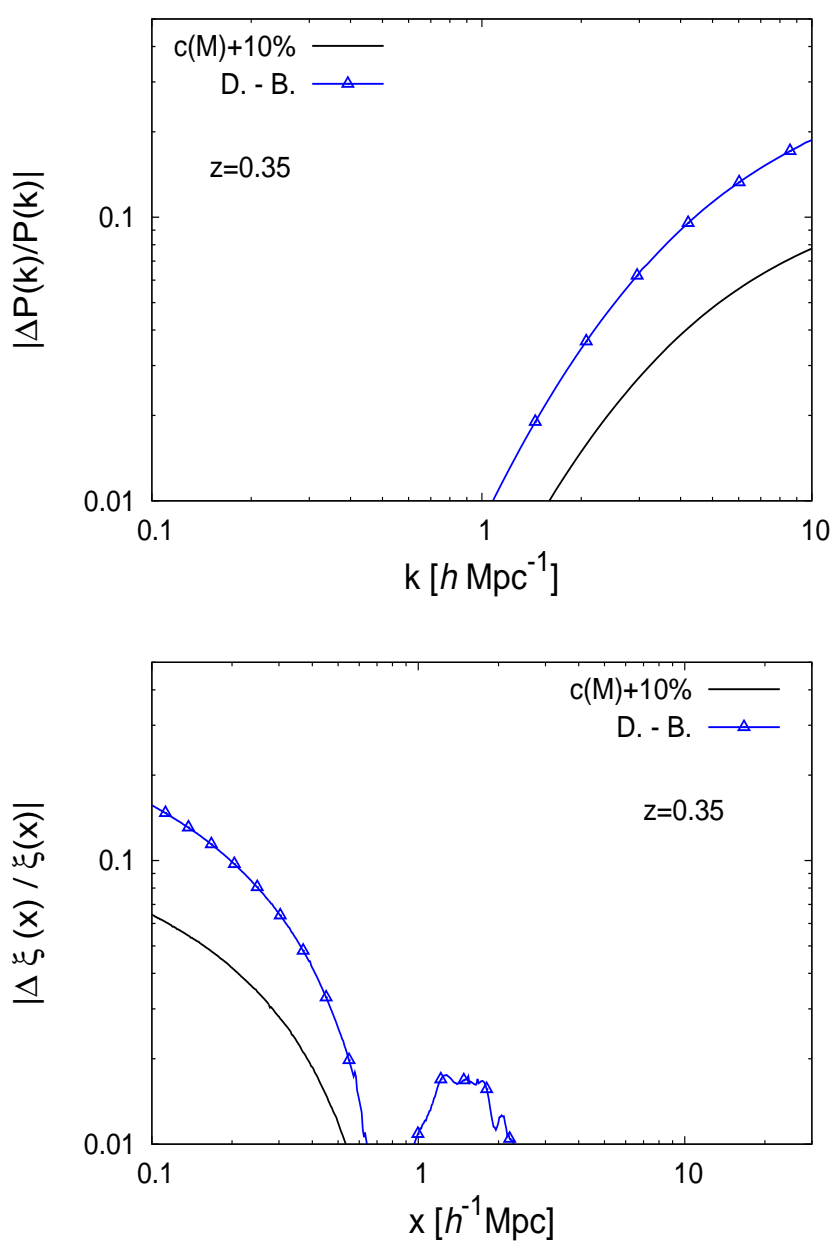

FIG. 10: Upper panel: relative change of the power spectrum at $z=0.35$ when the concentration parameter $c(M)$ is increased by $10 \%$, or when we change from the fit given in Ref. 31] to the one of Ref. 32] (label "D.-B." with triangle symbols). Lower panel: relative change of the correlation function at $z=0.35$ for the same cases.

function and density profiles. Indeed, because there is little hope that such properties can be predicted with a high accuracy by analytical models, they must be taken from numerical simulations. Then, the theoretical predictions become limited by the accuracy of these simulations, and more generally by these parameters; even more so when one considers different cosmologies than those where they were measured. This yields a limitation to the accuracy of semianalytical models but also of predictions that can be directly obtained from the numerical simulations themselves.

\section{Impact of the halo mass-concentration relation}

The halo density profiles are a first limitation to the accuracy of semianalytical models. Indeed, being the result
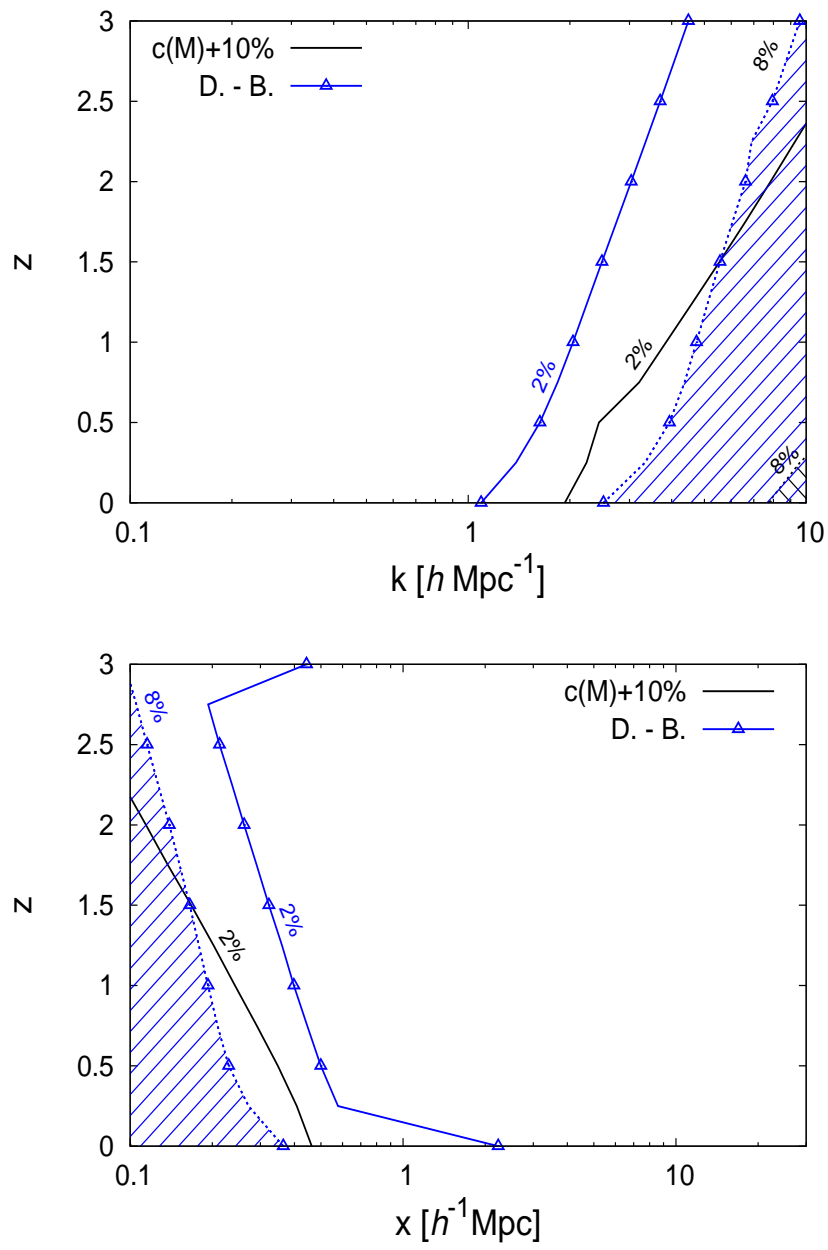

FIG. 11: Upper panel: contour lines in the $(k, z)$-plane of the regions where the power spectrum is modified by more than $2 \%$ (solid lines), or $8 \%$ (dotted lines), by a $10 \%$ increase of $c(M)$ or by changing from Ref. 31] to Ref. 32] (label "D.-B." with triangle symbols). Lower panel: similar contour lines in the $(x, z)$-plane for the two-point correlation.

of highly nonlinear and nonperturbative processes, it has proved difficult to obtain accurate and systematic theoretical predictions (especially within the virial radius). In practice one uses a typical fitting profile (e.g., a rational function) with a few parameters that may depend on the halo mass. Here, as in [17], we use the NFW profile 24. The exponents are fixed and there is a single concentration parameter, $c(M)$, that determines the transition scale between the inner and outer regimes $\rho \propto x^{-1}$ and $\rho \propto x^{-3}$.

We show in Fig. 10 the impact on the power spectrum and correlation function, at $z=0.35$, of a $10 \%$ increase of $c(M)$. We also show the difference between the predictions obtained using two different fits to numerical simulations from previous works, Refs. 31] and 32. We can see that the difference between published 
fits for $c(M)$ is of order $10 \%$ (somewhat greater; this also depends on mass and redshift). However, it appears that the impact on the power spectrum and correlation function is restricted to rather small scales, $k \gtrsim 1 h \mathrm{Mpc}^{-1}$ and $x \lesssim 2 h^{-1} \mathrm{Mpc}$ at $z=0.35$, for a $1 \%$ accuracy.

The contour lines in the $(k, z)$ and $(x, z)$ planes of a $2 \%$ or $8 \%$ impact on the power spectrum and correlation function are shown in Fig. 11, for these same modifications to $c(M)$. Again, we find that for the redshift range $0 \leq z \leq 3$ the uncertainties of the mass-concentration relation only affect the power spectrum and correlation function on rather small scales. The comparison with Fig. 9 shows that this occurs in the highly nonlinear regime where the perturbative expansions are no longer valid and the power spectrum or correlation function is dominated by the one-halo contribution.

Thus, the precise shape of halo profiles should not be a worrying limitation of semianalytical models, because there remains a large range of scales where its impact is negligible.

\section{Impact of the halo mass function}

Apart from the halo profiles, a second limitation to the accuracy of semianalytical models is the halo mass function itself. In principle, it should be more easily predicted than halo profiles, because one does not need to follow the late virialization stages of inner halo regions but only to count collapsed regions. This explains the relative success of various analytical approaches [36, 37] that try to detect future halos from the initial linear density field (for the high-mass tail). However, it has proved difficult to go below a 20\% accuracy (this depends on mass and redshift) and most works use fits to numerical simulations, or involve some parameters that are taken from simulations.

We show in Fig. 12 the impact on the power spectrum and correlation function, at $z=0.35$, of a $10 \%$ decrease of the mass function $n(M)$. We also show the difference between the predictions obtained using three different fits to numerical simulations, Refs. 33], 34], and 35]. We can see that the difference between published fits for $n(M)$ is of order $10 \%$ (somewhat greater; this also depends on mass and redshift). The comparison with Fig. 10 shows that the impact of a 10\% inaccuracy of the halo mass function is greater than the impact of a $10 \%$ inaccuracy of the mass-concentration relation. Therefore, this could be the limiting factor of semianalytical models.

Decreasing (or increasing) the halo mass function by $10 \%$ is not realistic because the total halo mass fraction should remain at unity (or at least not greater than unity). However, the power spectrum and correlation functions on large scales are mostly sensitive to massive and large halos, so that the constraint of a unit normalization (which is satisfied by the three fits from Refs. $33-$ [35]) is not sufficient to lessen the impact on the power spectrum. The large-mass tail is also difficult to measure
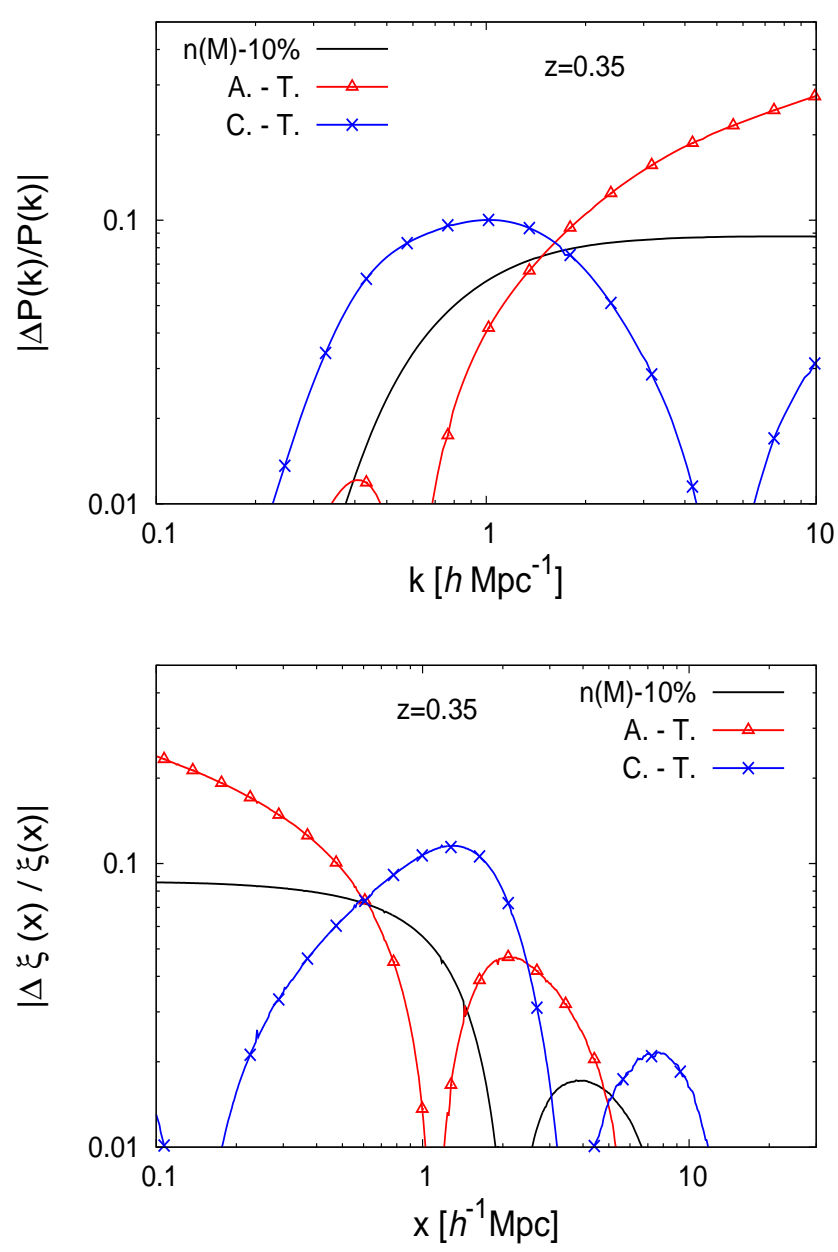

FIG. 12: Upper panel: relative change of the power spectrum at $z=0.35$ when the halo mass function $n(M)$ is decreased by $10 \%$, or when we change from the fit given in Ref. 33. to those of Ref. 34] (label "A.-T." with triangle symbols) or Ref. 35] (label "C.-T." with cross symbols). Lower panel: relative change of the correlation function at $z=0.35$ for the same cases.

from numerical simulations, because these are rare objects. These fits for halo mass functions are actually defined in different manners, as one can use different halofinder algorithms (e.g., based on a spherical-overdensity criterion or friends-of-friends procedures) and different halo definitions (e.g., different halo density contrasts). This is a further difficulty for semianalytical models, as different definitions may be relevant for different purposes.

In any case, Fig. 12 shows that there remains a significant range of scales that is not affected by these inaccuracies of the halo mass function. In particular, for the correlation function at $z=0.35$ scales beyond $10 h^{-1} \mathrm{Mpc}$ are not affected at the percent level. This means that accurate theoretical predictions can be obtained for a useful range of scales and redshifts. 

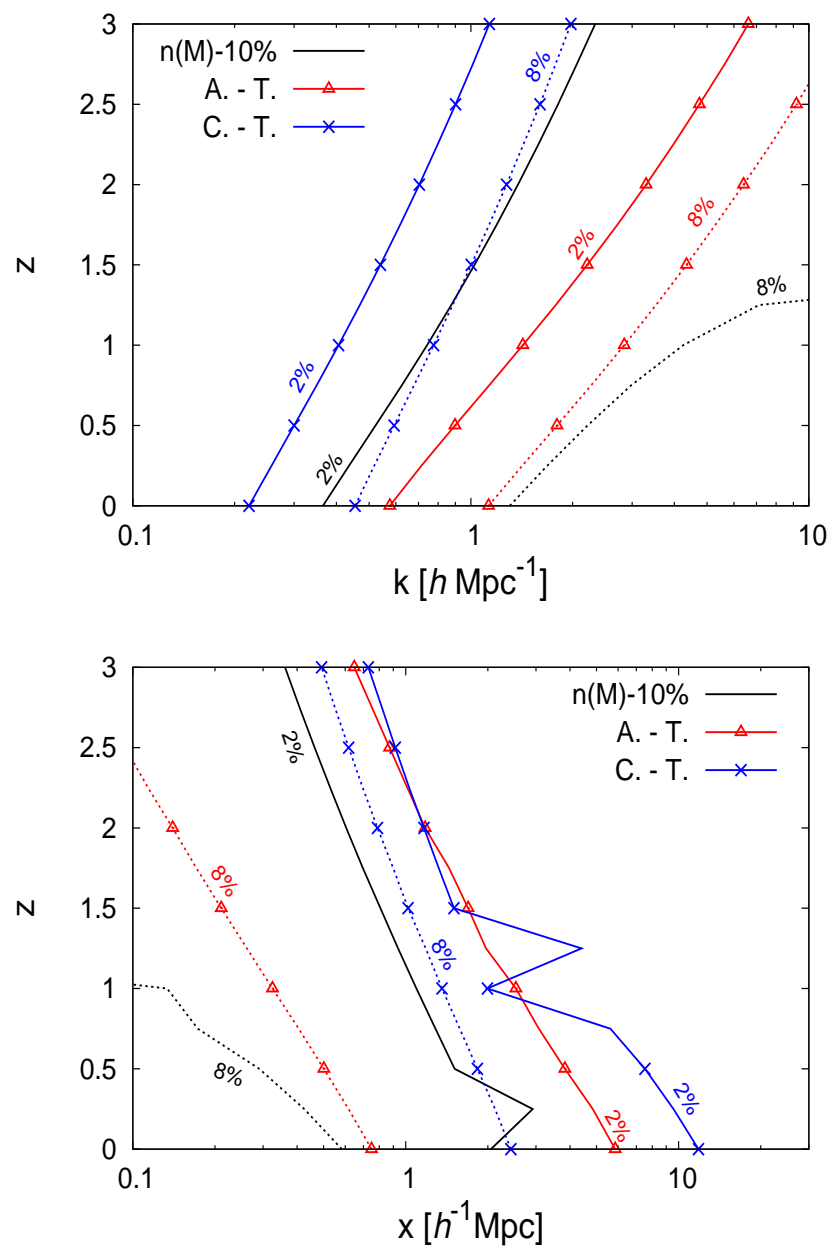

FIG. 13: Upper panel: contour lines in the $(k, z)$-plane of the regions where the power spectrum is modified by more than $2 \%$ (solid lines), or $8 \%$ (dotted lines), by a $10 \%$ decrease of $n(M)$, or by changing from Ref. 33] to Ref. 34] (label "A.T." with triangle symbols) or to Ref. 35] (label "C.-T." with cross symbols). Lower panel: similar contour lines in the $(x, z)$-plane for the two-point correlation.

The contour lines in the $(k, z)$ and $(x, z)$ planes of a $2 \%$ or $8 \%$ impact on the power spectrum and correlation function are shown in Fig. 13, for these same modifications to $n(M)$. We can check from the comparison with Fig. 9 that these effects only occur on small scales where the nonperturbative contributions are significant. Again, the comparison with Fig. 11 shows that in the redshift range $0 \leq z \leq 3$ the uncertainties of the halo mass function have a greater impact than those of the mass-concentration relationship. The real-space correlation function again appears to be more robust and provides a cleaner separation from these effects than the power spectrum.

The sources of uncertainty displayed in Figs. 12 and 13 also apply to the power spectra and correlation functions directly measured from numerical simulations. Our anal- ysis describes how the measures of halo mass functions and power spectra from simulations are correlated.

\section{CONCLUSION}

In this paper, using an accurate description of the matter power spectrum that combines perturbation theory with a halo model, we have investigated the possible accuracy that can be expected from semianalytical models.

First, focusing on the perturbative component, we have found that the simple reorganization of the standard perturbation theory with a Gaussian damping prefactor provides a well-ordered convergence for the power spectrum at low $k$. It also provides a finite two-point correlation function that is accurate at the percent level on BAO scales as soon as we go up to order $P_{L}^{2}$. Lagrangianspace expansions appear more efficient than their Eulerian counterparts when both are truncated at a low order, $N \leq 4$, but at high orders the convergence is no longer well ordered and shows signs of divergence at high $k$. On the other hand, the correlation function obtained from these Lagrangian-space expansions is also accurate at the percent level on BAO scales as soon as we go up to order $P_{L}^{2}$ (and $N \lesssim 4$ ).

Second, we have investigated the importance of nonperturbative contributions to the power spectrum. Those coming from the two-halo term, which may be seen as a backreaction of small scales onto large scales, affect a rather large range of wave numbers if one looks for a percent accuracy for $P(k)$. Those coming from the onehalo term, which correspond to inner halo regions, are restricted to higher wave numbers although they already reach a percent level at $k \sim 2 h \mathrm{Mpc}^{-1}$ at $z=0.35$. The separation between perturbative and nonperturbative effects appears to be better defined in configuration space. Thus, all nonperturbative effects are smaller than $1 \%$ at all redshifts on scales $x \gtrsim 10 h^{-1} \mathrm{Mpc}$, for the correlation function. This may be understood from the fact that these nonperturbative processes (shell crossing and virialization within halos) occur through local processes in real space, rather than wave interactions in Fourier space.

These estimates of the scales where nonperturbative effects are non-negligible can also be useful when one compares perturbative schemes with numerical simulations, to avoid meaningless comparisons. Indeed, whereas most perturbative schemes do not include shell-crossing effects, numerical simulations include all contributions at once and a good agreement on scales where the latter are not negligible can be misleading.

The relative importance of such nonperturbative effects is not necessarily a limit to semianalytical models if they can be accurately described. To assess the actual accuracy of semianalytical modeling, we have then estimated the impact on the power spectrum of the uncertainty of the mass-concentration relation and of the halo mass function. These uncertainties apply as well to the predictions obtained from the numerical simula- 
tions themselves. We find that the current accuracy of the mass-concentration relation (of order 10\%) is not a worrying limitation to theoretical predictions, as it only yields an uncertainty below the percent level on large scales with $k \lesssim 1 h \mathrm{Mpc}^{-1}$ or $x \gtrsim 1 h^{-1} \mathrm{Mpc}$ at all redshifts. This is due to the fact that changes to halo profiles only make a small-scale redistribution of matter and do not modify large-scale properties. The current uncertainty on the halo mass function is a greater problem for semianalytical models as it can affect wave numbers down to $k \sim 0.2 h \mathrm{Mpc}^{-1}$ and scales up to $x \sim 10 h^{-1} \mathrm{Mpc}$ at $z=0$ if we require a percent accuracy. Indeed, this corresponds to a reorganization of matter on the scale of the largest halos, because weakly nonlinear scales are mostly sensitive to the largest halos and the constraint associated with the normalization to unity of the halo mass function is not sufficient to damp this effect. Again, it appears that configuration-space statistics are better suited to separate such effects. In particular, while $k \sim 0.2 h \mathrm{Mpc}^{-1}$ is close to the scales $\left(k \sim 0.1 h \mathrm{Mpc}^{-1}\right)$ where baryon acoustic oscillations can be measured in the power spectrum, $x \sim 10 h^{-1} \mathrm{Mpc}$ is quite far from the correlation-function acoustic peak $\left(x \sim 105 h^{-1} \mathrm{Mpc}\right)$.

These results also apply to the power spectra measured in numerical simulations, as they describe how uncertainties of the halo mass functions and power spectra measured in these simulations are correlated.

From the observational point of view, one must then balance the higher accuracy of the theoretical predictions in configuration space with the easier handling of Fourierspace data, because of their better-behaved covariance matrices (which are diagonal in the linear regime because different wave numbers are uncorrelated).

\section{Acknowledgments}

This work is supported in part by the French Agence Nationale de la Recherche under Grant ANR-12-BS050002 .

\section{Appendix A: Appendix: Combining the halo model with one-loop perturbation theory}

In this appendix we provide some more details about the combination of one-loop perturbation theory and halo model that defines the nonlinear power spectrum that we use in this paper, given by Eqs.(1)-(3). See Ref. [17] for details.

In the Lagrangian-space framework, particles follow trajectories $\mathbf{x}_{i}(t)=\mathbf{q}_{i}+\Psi_{i}(t)$, where $\mathbf{q}_{i}$ is the initial position and $\Psi_{i}$ the displacement field. At linear order, the variances of the relative displacement $\Psi=\Psi_{2}-\Psi_{1}$ of two particles 1 and 2 , in the transverse and longitudinal directions with respect to the initial separation vector

$$
\begin{aligned}
& \mathbf{q}=\mathbf{q}_{2}-\mathbf{q}_{1}, \text { are } \\
& \sigma_{\|}^{2}(q)=\left\langle\Psi_{L \|}^{2}\right\rangle=2 \int \mathrm{d} \mathbf{k}\left[1-\cos \left(k_{\|} q\right)\right] \frac{k_{\|}^{2}}{k^{4}} P_{L}(k), \\
& \sigma_{\perp}^{2}(q)=\left\langle\Psi_{L \perp}^{2}\right\rangle=2 \int \mathrm{d} \mathbf{k}\left[1-\cos \left(k_{\|} q\right)\right] \frac{k_{\perp}^{2}}{k^{4}} P_{L}(k),
\end{aligned}
$$

where $k_{\perp}$ is the component along one of the two transverse directions. The power spectrum in the Zel'dovich approximation is obtained by considering the displacement field at linear order. For Gaussian initial conditions, this yields a Gaussian distribution for the relative displacements and, using the exact expression (15), this leads to the Zel'dovich power spectrum (16), where $\mu=(\mathbf{k} \cdot \mathbf{q}) /(k q)$. We go beyond the Zel'dovich approximation by including nonlinearities in the distribution of the parallel displacement field, while keeping linear theory for the transverse one. Thus, introducing the rescaled longitudinal relative displacement $\kappa$ of the pair of particles, and its linear variance $\sigma_{\kappa}^{2}$,

$$
\kappa=\frac{x_{\|}}{q}, \quad \sigma_{\kappa}^{2}=\frac{\sigma_{\|}^{2}}{q^{2}},
$$

we define its cumulant generating function $\varphi(y)$ by

$$
\left\langle e^{-y \kappa / \sigma^{2}}\right\rangle=e^{-\varphi(y) / \sigma_{\kappa}^{2}}
$$

By definition, this generating function must satisfy the series expansion (4), with the first few orders given by Eq.(5). Then, we use the ansatz

$$
\varphi(y)=\frac{1-\alpha}{\alpha}\left(1+\frac{y}{1-\alpha}\right)^{\alpha}-\frac{1-\alpha}{\alpha},
$$

where the scale-dependent parameter $\alpha(q)$ is given by

$$
\alpha(q)=\frac{2-S_{3}^{\kappa}(q)}{1-S_{3}^{\kappa}(q)} .
$$

This is the simplest function that ensures consistency with the constraint (5) [and that $-\varphi(y)$ be convex, which must be satisfied to provide a meaningful cumulant generating function]. The associated power spectrum $P_{\text {pert. }}(k)$ is given by Eq. (6), using Eq. (A4) into Eq. (14) and recalling that we keep linear theory for the transverse displacement. Moreover, $P_{\text {pert. }}(k)$ is exact up to one-loop order (i.e., up to order $\left.P_{L}^{2}\right)$ provided $S_{3}^{\kappa}(q)$ is given by

$$
\begin{aligned}
S_{3}^{\kappa}(q)= & -\frac{24 \pi}{\sigma_{\kappa}^{4}} \int_{0}^{\infty} \mathrm{d} k \frac{P^{1 \text { loop }}(k)-P_{\mathrm{Z}}^{1 \text { loop }}(k)}{q^{4} k^{2}} \\
& \times\left[2+\cos (k q)-3 \frac{\sin (k q)}{k q}\right]
\end{aligned}
$$

where $P^{\text {1loop }}(k)$ is the exact one-loop power spectrum constructed with standard perturbation theory, whereas 
$P_{\mathrm{Z}}^{1 \text { loop }}(k)$ is the one-loop power spectrum obtained from the Zel'dovich power spectrum (16). Thus, the power spectrum (6) is a generalization of the Zel'dovich power spectrum. It is consistent with the exact perturbative expansion up to one-loop order (i.e., $P_{L}^{2}$ ), whereas the Zel'dovich power spectrum only agrees at linear order, and it also contains some perturbative terms at all higher orders in both Eulerian and Lagrangian spaces [generated through the nonpolynomial function $\varphi(y)$ and the exponential in Eq.(6)].

The generating function $\varphi(y)$ of Eq. A4 also defines the probability distribution function of $\kappa$,

$$
\mathcal{P}_{\varphi}(\kappa)=\int_{-\mathrm{i} \infty}^{\mathrm{i} \infty} \frac{\mathrm{d} y}{2 \pi \mathrm{i} \sigma_{\kappa}^{2}} e^{[\kappa y-\varphi(y)] / \sigma_{\kappa}^{2}} .
$$

The perturbative expression (6) does not take into account nonperturbative phenomena such as shell crossings, which can be approximated using a simplified adhesion model whereby particles coalesce when $\kappa<0$. This is described by modifying the probability distribution as

$$
\mathcal{P}^{\text {ad. }}(\kappa)=a_{1} \Theta(\kappa>0) \mathcal{P}_{\varphi}(\kappa)+a_{0} \delta_{D}(\kappa),
$$

where $a_{0,1}$ are determined by the constraints $\langle 1\rangle=\langle\kappa\rangle=$ 1. This provides a simplified account of the formation of pancakes (the first nonperturbative structures on large scales, such as the "walls" around cosmic voids or underdense regions), and it leads to the "cosmic web" power spectrum

$$
\begin{gathered}
P_{\text {c.w. }}(k)=\int \frac{\mathrm{d} \mathbf{q}}{(2 \pi)^{3}} \frac{1}{1+A_{1}} e^{-\frac{1}{2} k^{2}\left(1-\mu^{2}\right) \sigma_{\perp}^{2}}\left\{e^{-\varphi\left(-\mathrm{i} k q \mu \sigma_{\kappa}^{2}\right) / \sigma_{\kappa}^{2}}\right. \\
\left.+A_{1}+\int_{0^{+}-\mathrm{i} \infty}^{0^{+}+\mathrm{i} \infty} \frac{\mathrm{d} y}{2 \pi \mathrm{i}} e^{-\varphi(y) / \sigma_{\kappa}^{2}}\left(\frac{1}{y}-\frac{1}{y+\mathrm{i} k q \mu \sigma_{\kappa}^{2}}\right)\right\},
\end{gathered}
$$

where $A_{1}=\left(1-a_{1}\right) / a_{1}$ is given by

$$
A_{1}=\sigma_{\kappa}^{2} \int_{0^{+-i} \infty}^{0^{+}+\mathrm{i} \infty} \frac{\mathrm{d} y}{2 \pi \mathrm{i} y^{2}} e^{-\varphi(y) / \sigma_{\kappa}^{2}} .
$$

The power spectra (6) and (A10) are identical to all orders of perturbation theory, and only differ by nonperturbative corrections of the form $e^{-1 / \sigma^{2}}$ associated with the adhesionlike modification (A9).
To go to highly nonlinear scales, we use the halo model and the power spectrum is split over one-halo and twohalo components as in Eq.(1). Then, the probability that two particles of initial separation $q$ belong to the same halo of mass $M$ is 38]

$$
F_{1 \mathrm{H}}(q)=\int_{\nu_{q / 2}}^{\infty} \frac{d \nu}{\nu} f(\nu) \frac{\left(2 q_{M}-q\right)^{2}\left(4 q_{M}+q\right)}{16 q_{M}^{3}},
$$

where $f(\nu)$ is the scaling function that determines the halo mass function, $\nu=\delta_{L}(M) / \sigma_{M}$, and $M=4 \pi \bar{\rho} q_{M}^{3} / 3$. (The lower bound of the integral corresponds to the mass enclosed within a radius $q / 2$.) The probability of belonging to two halos is $F_{2 \mathrm{H}}=1-F_{1 \mathrm{H}}$. Finally, the average of the component of the particle displacements that is associated with small-scale virialized motions within halos reads as

$$
\left\langle e^{\mathrm{ik} \cdot \mathbf{x}}\right\rangle_{q}^{\mathrm{vir}}=\left[\frac{\int_{0}^{\nu_{q / 2}} \frac{d \nu}{\nu} f(\nu) \tilde{u}_{M}(k)}{\int_{0}^{\nu_{q / 2}} \frac{d \nu}{\nu} f(\nu)}\right]^{2},
$$

because we assume that virialized motions within two different halos are uncorrelated. Here, we have defined the Fourier transform of the halo profile as

$$
\tilde{u}_{M}(k)=\frac{\int \mathrm{d} \mathbf{x} e^{-i \mathbf{k} \cdot \mathbf{x}} \rho_{M}(x)}{\int \mathrm{d} \mathbf{x} \rho_{M}(x)},
$$

with $M=\int \mathrm{d} \mathbf{x} \rho_{M}(x)$.

Then, the two-halo part $P_{2 \mathrm{H}}(k)$ of the power spectrum is given by Eq.(3), where we recognize the "cosmic web" power spectrum (A10), to which we have added the factor $F_{2 \mathrm{H}}$, to avoid double counting with the one-halo term, and the small-scale motions factor A13, to take into account the finite width of halos. The one-halo part $P_{1 \mathrm{H}}(k)$ is given as usual by Eq.(2), with the counterterm $\tilde{W}^{2}$ associated with mass and momentum conservation, which ensures that $P_{1 \mathrm{H}}(k) \propto k^{4}$ at low $k$. Again, this gives a "halo-model" power spectrum (11) that is identical to Eq.(6) at all orders of perturbation theory. In particular, thanks to the choice (A7), it agrees with standard perturbation theory up to one-loop order (and contains partial terms at all higher orders, generated through the function $\varphi(y)$, as well as nonperturbative terms of the form $\left.e^{-1 / \sigma^{2}}\right)$.
[1] P. J. E. Peebles, The large-scale structure of the universe (Princeton University Press, Princeton, N.J., USA, 1980).

[2] R. Laureijs, J. Amiaux, S. Arduini, J. . Auguères, J. Brinchmann, R. Cole, M. Cropper, C. Dabin, L. Duvet, A. Ealet, et al., arXiv:1110.3193 (2011), 1110.3193.

[3] A. Cooray and R. Sheth, Phys. Rep. 372, 1 (2002), astro$\mathrm{ph} / 0206508$.
[4] D. J. Eisenstein and W. Hu, Astrophys. J. 496, 605 (1998), arXiv:astro-ph/9709112.

[5] D. J. Eisenstein, I. Zehavi, D. W. Hogg, R. Scoccimarro, M. R. Blanton, R. C. Nichol, R. Scranton, H.-J. Seo, M. Tegmark, Z. Zheng, et al., Astrophys. J. 633, 560 (2005), arXiv:astro-ph/0501171.

[6] M. H. Goroff, B. Grinstein, S.-J. Rey, and M. B. Wise, Astrophys. J. 311, 6 (1986). 
[7] F. Bernardeau, S. Colombi, E. Gaztañaga, and R. Scoccimarro, Phys. Rep. 367, 1 (2002), arXiv:astro$\mathrm{ph} / 0112551$.

[8] M. Crocce and R. Scoccimarro, Phys. Rev. D 73, 063519 (2006), arXiv:astro-ph/0509418.

[9] M. Crocce and R. Scoccimarro, Phys. Rev. D 73, 063520 (2006), arXiv:astro-ph/0509419.

[10] P. Valageas, Astr. \& Astrophys. 465, 725 (2007), arXiv:astro-ph/0611849.

[11] A. Taruya and T. Hiramatsu, Astrophys. J. 674, 617 (2008), 0708.1367.

[12] M. Pietroni, JCAP 10, 36 (2008), 0806.0971.

[13] A. Taruya, F. Bernardeau, T. Nishimichi, and S. Codis, Phys. Rev. D 86, 103528 (2012), 1208.1191.

[14] S. Anselmi and M. Pietroni, JCAP 12, 013 (2012), 1205.2235 .

[15] T. Matsubara, Phys. Rev. D 77, 063530 (2008), 0711.2521.

[16] T. Matsubara, Phys. Rev. D 78, 083519 (2008), 0807.1733.

[17] P. Valageas, T. Nishimichi, and A. Taruya, Phys. Rev. D 87, 083522 (2013), 1302.4533.

[18] M. Pietroni, G. Mangano, N. Saviano, and M. Viel, JCAP 1, 019 (2012), 1108.5203.

[19] J. J. M. Carrasco, M. P. Hertzberg, and L. Senatore, Journal of High Energy Physics 9, 82 (2012), 1206.2926.

[20] N. Afshordi, Phys. Rev. D 75, 021302 (2007), arXiv:astro-ph/0610336.

[21] P. Valageas, Astr. \& Astrophys. 526, A67+ (2011), 1009.0106.

[22] Y. B. Zel'Dovich, Astr. 63 Astrophys. 5, 84 (1970).

[23] S. Pueblas and R. Scoccimarro, Phys. Rev. D 80, 043504 (2009), 0809.4606.
[24] J. F. Navarro, C. S. Frenk, and S. D. M. White, Astrophys. J. 490, 493 (1997), arXiv:astro-ph/9611107.

[25] P. J. E. Peebles, Astr. \& Astrophys. 32, 391 (1974).

[26] P. Schneider and M. Bartelmann, Mon. Not. R. Astr. Soc. 273, 475 (1995).

[27] P. Valageas, Astr. \& Astrophys. 476, 31 (2007), 0706.2593.

[28] P. Valageas, Astr. \& Astrophys. 484, 79 (2008), 0711.3407.

[29] A. N. Taylor and A. J. S. Hamilton, Mon. Not. R. Astr. Soc. 282, 767 (1996), arXiv:astro-ph/9604020.

[30] J. Carlson, B. Reid, and M. White, Mon. Not. R. Astr. Soc. 429, 1674 (2013), 1209.0780.

[31] A. R. Duffy, J. Schaye, S. T. Kay, and C. Dalla Vecchia, Mon. Not. R. Astr. Soc. 390, L64 (2008), 0804.2486.

[32] S. Bhattacharya, S. Habib, K. Heitmann, and A. Vikhlinin, Astrophys. J. 766, 32 (2013), 1112.5479.

[33] J. Tinker, A. V. Kravtsov, A. Klypin, K. Abazajian, M. Warren, G. Yepes, S. Gottlöber, and D. E. Holz, Astrophys. J. 688, 709 (2008), 0803.2706.

[34] R. E. Angulo, V. Springel, S. D. M. White, A. Jenkins, C. M. Baugh, and C. S. Frenk, Mon. Not. R. Astr. Soc. 426, 2046 (2012), 1203.3216.

[35] J. Courtin, Y. Rasera, J.-M. Alimi, P.-S. Corasaniti, V. Boucher, and A. Füzfa, Mon. Not. R. Astr. Soc. 410, 1911 (2011), 1001.3425.

[36] W. H. Press and P. Schechter, Astrophys. J. 187, 425 (1974).

[37] A. Paranjape, R. K. Sheth, and V. Desjacques, Mon. Not. R. Astr. Soc. 431, 1503 (2013), 1210.1483.

[38] P. Valageas and T. Nishimichi, Astr. Es Astrophys. 527, A87+ (2011), 1009.0597. 